\title{
Lorentzian vacuum transitions for anisotropic universes
}

\author{
H. García-Compeán $\odot^{*}$ and D. Mata-Pacheco $\odot^{\dagger}$ \\ Departamento de Física, Centro de Investigación y de Estudios Avanzados del IPN, \\ P.O. Box 14-740, CP. 07000, Ciudad de México, México
}

(Received 22 July 2021; accepted 4 October 2021; published 22 November 2021)

\begin{abstract}
The vacuum transition probabilities for anisotropic universes in the presence of a scalar field potential in the Wentzel-Kramers-Brillouin approximation are studied. We follow the work by Cespedes et al. [Phys. Rev. D 104, 026013 (2021)], which discuss these transitions in the isotropic context using the WheelerDeWitt equation, the Lorentzian Hamiltonian approach and the thin wall limit. First, we propose a general procedure to adapt their formalism to compute the decay rates for any superspace model. Then we apply it to compute the transition probabilities of an Friedmann-Lemaitre-Robertson-Walker (FLRW) metric with both positive and zero curvature, reproducing in this way one of the results obtained at Cespedes et al. We then proceed to apply the formalism to three anisotropic metrics, namely, Kantowski-Sachs, Bianchi III and biaxial Bianchi IX to compute the rate decays for these three cases. In the process we find that this method involves some conditions which relates the effective number of independent degrees of freedom resulting on all probabilities being described with only two independent variables. For the Bianchi III metric, we find that a general effect of anisotropy is to decrease the transition probability as the degree of anisotropy is increased, having as the isotropic limit the flat FLRW result.
\end{abstract}

DOI: $10.1103 /$ PhysRevD.104.106014

\section{INTRODUCTION}

For some decades the theory of quantum gravity has been one of the most important challenges of theoretical physics. Over the years, many approaches to this subject have been developed presenting a list of different efforts to achieve a consistent theory. One of the first attempts in this regard is the use of a Hamiltonian formalism and canonical quantization [1] to obtain a quantum picture of gravity leading to a Schrödinger-like equation, namely, the Wheeler-DeWitt (WDW) equation [2,3]. In this formalism there is not a time variable in which the wave functionals can depend on, leading to difficulties when interpreting the solutions (the problem of time). Nevertheless, the decay rate, i.e., the ratio of absolute squares of the wave functionals for different configurations can be interpreted as relative probabilities between two states in which the system performs a transition.

Cosmological problems of the early universe are a natural scenario in which the effects of such a quantum theory are expected to be of first importance. In particular,

\footnotetext{
*ompean@fis.cinvestav.mx

dmata@fis.cinvestav.mx
}

Published by the American Physical Society under the terms of the Creative Commons Attribution 4.0 International license. Further distribution of this work must maintain attribution to the author(s) and the published article's title, journal citation, and DOI. Funded by SCOAP ${ }^{3}$. there has been great interest over the years in the study of effects of vacuum decay in this type of theories. As in field theory, this process is described by the nucleation of true vacuum bubbles. Euclidean methods to study these processes were developed originally by Sidney Coleman and collaborators for field theory $[4,5]$ and then, they were extended to include gravity by Coleman and De Luccia [6]. Their results were later generalized by Parke [7]. Recently a Hamiltonian approach to study vacuum transitions without using Euclidean methods was developed in Refs. [8,9]. The Fischler, Morgan and Polchinski (FMP) procedure was used in $[10,11]$ to compute tunneling probabilities of transitions between two de Sitter universes and the transition from Minkowski to de Sitter is also possible without having singular instantons. A generalization of FMP method to include explicitly scalar field potentials for the general Wheeler's superpace and using a WentzelKramers-Brillouin (WKB) approximation was developed in the work [11]. Although the method can be defined on the general superspace, in order to compute some concrete observable, for simplicity one usually is restricted to minisuperspace. The method is applicable to any number of fields and with the additional thin wall limit, it produces the same results for a closed Friedmann-LemaitreRobertson-Walker (FLRW) metric as the one obtained by the uses of Euclidean methods [10,11].

On the other hand, many different studies based in satellite observations have been carried out regarding the deviations of the cosmological isotropy. For instance 
analysis from the cosmic microwave background (CMB) measures of temperature and polarization from Planck observatory [12], seems to strongly imply that the deviations from isotropy are disfavored. However other observations on type Ia supernovae of the cosmic acceleration [13] seems to imply certain degree of anisotropy which may play a fundamental role in the cosmological models. Although a more recent work on this subject challenges this assertion and gives support to statistical isotropy [14]. Other groups have studied the anisotropy using basedspace X-ray observations and have found some results compatible with a nonvanishing degree of large scale anisotropy [15]. Moreover, some implications of the anisotropy in galaxy clusters is analyzed in [16]. More recently, the study of the tension in the value of the Hubble constant would imply a modification of the current paradigm Einstein theory + FLRW Universe. The presence of anisotropies seem to be compatible with those results $[17,18]$. Then the debate about the possible existence of a certain degree of anisotropy is still not conclusive when all observations are taken into account. Thus the importance of studying homogeneous but anisotropic universes in the context of quantum gravity is quite relevant and not empty. Vacuum transitions for these types of universes have also been studied previously. In reference [19], it was described these transitions involving only gravity and a cosmological constant for the Bianchi IX model. In the mentioned work, these transitions have also been studied in the presence of a scalar field. Moreover, in Ref. [20], it is described these transitions for a Kantowski-Sachs metric using also Euclidean methods and in reference [21], the Bianchi IX model is studied in the tunneling from nothing scenario. Finally, in reference [22] transitions for two minima of a scalar field potential for the Bianchi I model was also studied into this context.

In the present article we study vacuum transitions using the Hamiltonian formalism developed in [11] for three anisotropic metrics: (a) Kantowski-Sachs, (b) Bianchi III and (c) the biaxial Bianchi IX. In particular, we focus on obtaining transition probabilities in the semi-classical approximation for two minima of the potential in a Lorentzian computation, without resorting to any Euclidean method.

This work is organized as follows. In Sec. II we give a brief review of the general proposal of Ref. [11]. It is also presented a general procedure to apply this method to any superspace model. In the next sections the procedure is applied to some examples in the minisuperspace. Section III is devoted to apply our method to the FLRW metric with positive curvature and we show that our procedure gives the same results as the original work, we also point out a particular remark. We apply our method to obtain the transition probabilities in the remaining sections for the FLRW isotropic and plane metric in Sec. IV and for the anisotropic metrics: Kantowski-Sachs in Sec. V, Bianchi III in Sec. VI and biaxial Bianchi IX in Sec. VII. Finally, in Sec. VIII we discuss our results.

\section{WKB APPROXIMATION FOR QUANTUM GRAVITY}

In this section we start by introducing the general approach proposed in Ref. [11], which applies the WKB approximation to the WDW equation. We follow the notation and conventions given at Ref. [11]. In particular, we stress our attention in obtaining a general expression for the semi-classical decay rate between a false and a true vacuum of a scalar field potential.

In the context of the ADM-formulation of general relativity (GR) [1-3] we know that the general form of the Hamiltonian constraint is given by

$$
\mathcal{H}=\frac{1}{2} G^{M N}(\Phi) \pi_{M} \pi_{N}+f[\Phi] \approx 0,
$$

where $G^{M N}$ is the inverse metric in Wheeler's superspace, $\Phi$ represents all the coordinates $\Phi^{M}$ of the superspace (which can be infinite dimensional), that is, the components of the three-dimensional metric, the matter field variables, etc., and their corresponding canonically conjugate momenta $\pi_{M}$. Moreover $f[\Phi]$ stands for all the other terms of 3-curvature and potential terms of matter fields that arise in the WDW equation. The WDW equation is obtained after doing a canonical quantization of the Hamiltonian constraint, that is, replacing $\pi_{M} \rightarrow-i \hbar \frac{\delta}{\delta \Phi^{M}}$ in the last expression, from where we obtain up to ordering ambiguities

$\mathcal{H} \Psi(\Phi)=\left[-\frac{\hbar^{2}}{2} G^{M N}(\Phi) \frac{\delta}{\delta \Phi^{M}} \frac{\delta}{\delta \Phi^{N}}+f[\Phi]\right] \Psi[\Phi]=0$,

where $\Psi[\Phi]$ is the usual wave functional in superspace.

In order to obtain a semiclassical result, we take the general proposal of the WKB type

$$
\Psi[\Phi]=\exp \left\{\frac{i}{\hbar} S[\Phi]\right\},
$$

with the $\hbar$-expansion

$$
S[\Phi]=S_{0}[\Phi]+\hbar S_{1}[\Phi]+\mathcal{O}\left(\hbar^{2}\right) .
$$

Substituting this in the WDW equation (2) we obtain for the two lowest order in $\hbar$

$$
\begin{gathered}
\frac{1}{2} G^{M N} \frac{\delta S_{0}}{\delta \Phi^{M}} \frac{\delta S_{0}}{\delta \Phi^{N}}+f[\Phi]=0, \\
2 G^{M N} \frac{\delta S_{0}}{\delta \Phi^{M}} \frac{\delta S_{1}}{\delta \Phi^{N}}=i G^{M N} \frac{\delta^{2}}{\delta \Phi^{M} \delta \Phi^{N}} S_{0} .
\end{gathered}
$$


Given a set of integral curves with parameter $s$ defined on a particular slice on the space of fields, they are described by

$$
C(s) \frac{d \Phi^{M}}{d s}=G^{M N} \frac{\delta S_{0}}{\delta \Phi^{N}} .
$$

We can write down the classical action in the form

$$
S_{0}\left[\Phi_{s}\right]=\int^{\Phi_{s}} \int_{X} \pi_{M} d x^{M},
$$

which, by using Eqs. (5) and (7), it can be rewritten as

$$
S_{0}\left[\Phi_{s}\right]=-2 \int \frac{d s^{\prime}}{C\left(s^{\prime}\right)} \int_{X} f\left[\Phi_{s^{\prime}}\right]
$$

Manipulating Eqs. (5) and (7) we find the following relation

$$
G_{M N} \frac{d \Phi^{M}}{d s} \frac{d \Phi^{N}}{d s}=-\frac{2 f\left[\Phi_{s}\right]}{C^{2}(s)},
$$

where $G_{M N}$ is the inverse of $G^{M N}$.

We can see that Eqs. (7) and (10) form a system of $n+1$ equations for the $n+1$ variables: $\left(\frac{d \Phi^{M}}{d s}, C^{2}(s)\right)$ which in principle can be solved and then substituted back into Eq. (9) to obtain the classical action. Therefore, in principle, the general setup presented provides us with enough information to compute the classical action, and thus the wave functional to first order in $\hbar$.

Let us assume that the fields $\Phi^{M}$ depend only on the time variable, then using Eq. (9), the variational derivative in (7) can be expressed in terms of a partial derivative of the function $f[\Phi]$. By doing this the system can be solved in general giving as solutions

$$
\begin{aligned}
C^{2}(s) & =-\frac{2 \operatorname{Vol}^{2}(X)}{f[\Phi]} G^{M N} \frac{\partial f}{\partial \Phi^{M}} \frac{\partial f}{\partial \Phi^{N}}, \\
\frac{d \Phi^{M}}{d s} & =\frac{f[\Phi]}{\operatorname{Vol}(X)} \frac{G^{M N} \frac{\partial f}{\partial \Phi^{N}}}{G^{L O} \frac{\partial f}{\partial \Phi^{L}} \frac{\partial f}{\partial \Phi^{O}}},
\end{aligned}
$$

where $\operatorname{Vol}(X)$ is the volume of the spatial slice. From Eq. (12) we see that in general, we can obtain the following system of differential equations relating the various fields

$$
\frac{d \Phi^{M}}{d \Phi^{N}}=\frac{G^{M L} \frac{\partial f}{\partial \Phi^{L}}}{G^{N P} \frac{\partial f}{\partial \Phi^{P}}}
$$

which is valid for every value of $M$ and $N$ such that $d \Phi^{M, N} \neq 0$. Therefore, the degrees of freedom are reduced in general and the classical action will involve less fields on its computation.
We now consider the matter content of the system to be only a scalar field canonically coupled to gravity and with a potential that has a false and a true vacuum. We study a wave functional corresponding to a path in field space in which the scalar field evolves from the false minimum to the true one, and one in which the system is kept at the false minimum during the path. Then the squared ratio of the absolute value of these wave functionals can be interpreted as the transition probability for the system to tunnel from the false to the true vacuum. Therefore, in the WKB approach considered here, the transition tunneling probability of going from the false vacuum at $\phi_{A}$ to the true vacuum at $\phi_{B}$ is the decay rate which is given by

$$
\begin{aligned}
P(A \rightarrow B) & =\left|\frac{\Psi\left(\varphi_{0}^{I}, \phi_{B} ; \varphi_{m}^{I}, \phi_{A}\right)}{\Psi\left(\varphi_{0}^{I}, \phi_{A} ; \varphi_{m}^{I}, \phi_{A}\right)}\right|^{2} \\
& =\left|\frac{\beta e^{\frac{i}{\hbar} S_{0}\left(\varphi_{0}^{I}, \phi_{B} ; \varphi_{m}^{I}, \phi_{A}\right)}+\chi e^{-\frac{i}{\hbar} S_{0}\left(\varphi_{0}^{I}, \phi_{B} ; \varphi_{m}^{I}, \phi_{A}\right)}}{\beta e^{\frac{i}{\hbar} S_{0}\left(\varphi_{0}^{I}, \phi_{A} ; \varphi_{m}^{I}, \phi_{A}\right)}+\chi e^{-\frac{i}{\hbar} S_{0}\left(\varphi_{0}^{I}, \phi_{A} ; \varphi_{m}^{I}, \phi_{A}\right)}}\right|^{2} \\
& =\left|e^{-\Gamma}\right|^{2},
\end{aligned}
$$

where we have denoted as $\varphi^{I}$ the remaining fields on superspace apart from the scalar degree of freedom, $\Psi\left(\varphi_{0}^{I}, \phi_{B}, \varphi_{m}^{I}, \phi_{A}\right)$ is the wave functional corresponding to the path that starts in $\varphi^{I}(s=0)=\varphi_{0}^{I}$ with the scalar field $\phi_{B}$ and ends in $\varphi^{I}\left(s=s_{M}\right)=\varphi_{m}^{I}$ with the scalar field $\phi_{A}$. In Eq. (14) $\beta$ and $\chi$ are the coefficients of the linear superposition to construct the general solution for the wave functionals. However, since all the terms involved are exponentials, we note that in each case one of the terms would be exponentially bigger than the other, and consequently, we can safely consider just the dominant term. Therefore, since we are working on a semiclassical approach, we will keep only the first term in the WKB expansion (4), $\Gamma$ will be given in any case as

$\pm \Gamma=\frac{i}{\hbar} S_{0}\left(\varphi_{0}^{M}, \phi_{B} ; \varphi_{m}^{M}, \phi_{A}\right)-\frac{i}{\hbar} S_{0}\left(\varphi_{0}^{M}, \phi_{A} ; \varphi_{m}^{M}, \phi_{A}\right)$,

where the \pm choice depends on which term is dominant in both the numerator and denominator above. Therefore, we have

$$
\begin{aligned}
P(A \rightarrow B)= & \exp [-2 \operatorname{Re}(\Gamma)] \\
= & \exp \left\{ \pm 2 \operatorname{Re}\left[\frac{i}{\hbar} S_{0}\left(\varphi_{0}^{M}, \phi_{B} ; \varphi_{m}^{M}, \phi_{A}\right)\right.\right. \\
& \left.\left.-\frac{i}{\hbar} S_{0}\left(\varphi_{0}^{M}, \phi_{A} ; \varphi_{m}^{M}, \phi_{A}\right)\right]\right\} .
\end{aligned}
$$

\section{TRANSITIONS IN FLRW WITH POSITIVE CURVATURE}

In the previous section the described formalism was valid in the Wheeler's superspace which has in general, an 
infinite number of variables. However, as it is well known, it is very complicated to perform a computation in this context. Thus, from now on we will work in minisuperspace which has a finite number of coordinates.

We start by studying the transitions in the case of a FLRW metric with positive curvature. The transition probability for this case was already obtained in [11] but we derive it again here using the general method described in the previous section and we mention a subtlety not considered in that work. The FLRW metric with positive curvature can be written as

$$
d s^{2}=-N^{2}(t) d t^{2}+a^{2}(t)\left(d r^{2}+\sin ^{2} \theta d \Omega_{2}^{2}\right),
$$

where $N(t)$ is the lapse function, $d \Omega_{2}^{2}$ is the metric of a 2sphere and $0 \leq r \leq \pi$. Considering gravity coupled to a scalar field that depends only on the time variable $\phi=\phi(t)$, and using natural units in which $c=1$ and $8 \pi G=1$, we obtain the Lagrangian for this system to be

$$
\begin{aligned}
\mathcal{L}= & 3 N(t) a(t)-\frac{3 a(t) \dot{a}(t)^{2}}{N(t)}+\frac{a^{3}(t) \dot{\phi}^{2}}{2 N(t)} \\
& -N(t) a^{3}(t) V(\phi),
\end{aligned}
$$

where $\dot{a}(t)$ stands for the derivative with respect to the time variable $t$ and $V(\phi)$ is the scalar field potential. Performing standard computations we obtain the canonical momenta

$$
\pi_{N}=0, \quad \pi_{a}=-\frac{6 a \dot{a}}{N}, \quad \pi_{\phi}=\frac{a^{3} \dot{\phi}}{N},
$$

and the Hamiltonian constraint

$$
H=N\left[\frac{\pi_{\phi}^{2}}{2 a^{3}}-\frac{\pi_{a}^{2}}{12 a}-3 a+a^{3} V\right] \approx 0 .
$$

Since the canonical momentum with respect to $N$ vanishes, we can ignore the prefactor and focus only on the term inside brackets in the last expression, then by comparing it with the general form of Eq. (1) we see that for this metric the set of variables is $\left\{\Phi^{M}\right\}=\{a, \phi\}$. While the nonvanishing elements of the metric in minisuperspace are

$$
G^{a a}=-\frac{1}{6 a}, \quad G^{\phi \phi}=\frac{1}{a^{3}},
$$

and

$$
f(a, \phi)=-3 a+a^{3} V(\phi) .
$$

We also note that in this case the volume of the spatial slice is given by
$\operatorname{Vol}(X)=\int_{\phi=0}^{2 \pi} \int_{\theta=0}^{\pi} \int_{r=0}^{\pi} \sin ^{2} r \sin \theta d r d \theta d \phi=2 \pi^{2}$,

which is evidently finite.

We choose the parameter $s$ such that for the interval $[0, \bar{s}-\delta s]$, where $s=0$ is the initial value, the field remains close to its value at the true minimum $\phi_{B}$, and for the interval $\left[\bar{s}+\delta s, s_{m}\right]$ the field remains very close to its value at the false minimum $\phi_{A}$, that is, we choose the parameter $s$ such that

$$
\phi(s) \approx \begin{cases}\phi_{B}, & 0<s<\bar{s}-\delta s \\ \phi_{A}, & \bar{s}+\delta s<s<s_{M} .\end{cases}
$$

Therefore, using Eq. (9) we have

$$
\begin{aligned}
S_{0}\left(a_{0}, \phi_{B} ; a_{m}, \phi_{A}\right)= & -4 \pi^{2} \int_{0}^{s_{M}} \frac{d s}{C(s)}\left[-3 a+a^{3} V(\phi)\right] \\
= & -4 \pi^{2}\left\{\int_{0}^{\bar{s}-\delta s} \frac{d s}{C(s)}\left[-3 a+a^{3} V_{B}\right]\right. \\
& +\int_{\bar{s}-\delta s}^{\bar{s}+\delta s} \frac{d s}{C(s)}\left[-3 a+a^{3} V(\phi)\right] \\
& \left.+\int_{\bar{s}+\delta s}^{s_{M}} \frac{d s}{C(s)}\left[-3 a+a^{3} V_{A}\right]\right\}, \quad
\end{aligned}
$$

where $V_{B}=V\left(\phi=\phi_{B}\right)$ and $V_{A}=V\left(\phi=\phi_{A}\right)$. We also have

$S_{0}\left(a_{0}, \phi_{A} ; a_{m}, \phi_{A}\right)=-4 \pi^{2} \int_{0}^{s_{M}} \frac{d s}{C(s)}\left[-3 a+a^{3} V_{A}\right]$

Substituting these expressions back in Eq. (15) we obtain

$$
\begin{aligned}
\pm \Gamma= & -\frac{4 \pi^{2} i}{\hbar} \int_{0}^{\bar{s}-\delta s} \frac{d s}{C(s)}\left[-3 a+a^{3} V_{B}\right] \\
& +\frac{4 \pi^{2} i}{\hbar} \int_{0}^{\bar{s}-\delta s} \frac{d s}{C(s)}\left[-3 a+a^{3} V_{A}\right] \\
& -\frac{4 \pi^{2} i}{\hbar} \int_{\bar{s}-\delta s}^{\bar{s}+\delta s} \frac{d s}{C(s)} a^{3}\left[V(\phi)-V_{A}\right] .
\end{aligned}
$$

In order to compute the two first integrals, we study the system of equations (7) and (10) for this metric in the particular case in which the scalar field is constant. The general solutions (11) and (12) take in this case the form

$$
\begin{aligned}
C^{2}(s) & =4 \pi^{4} \frac{\left(a V_{A, B}-\frac{1}{a}\right)^{2}}{\frac{a^{2}}{3} V_{A, B}-1}, \\
\frac{d a}{d s} & =\frac{1}{2 \pi^{2}} \frac{\frac{a^{2}}{3} V_{A, B}-1}{a V_{A, B}-\frac{1}{a}},
\end{aligned}
$$


where in these solutions $V_{A, B}$ represents either $V_{A}$ or $V_{B}$. Then, after a change of the integration variable from $s$ to $a$ according to $d s=\left(\frac{d a}{d s}\right)^{-1} d a$, we obtain

$$
-4 \pi^{2} \int \frac{d s}{C(s)}\left[-3 a+a^{3} V_{A, B}\right]= \pm 12 \pi^{2} i \int a\left(\sqrt{1-\frac{a^{2}}{3} V_{A, B}}\right) d a= \pm \frac{12 \pi^{2} i}{V_{A, B}}\left[1-\frac{V_{A, B}}{3} a^{2}\right]^{3 / 2} .
$$

Putting $a(\bar{s} \pm \delta s)=\bar{a} \pm \delta a$ we obtain

$$
-4 \pi^{2} i \int_{0}^{\bar{s}-\delta s} \frac{d s}{C(s)}\left[-3 a+a^{3} V_{A, B}\right]=\mp \frac{12 \pi^{2}}{V_{A, B}}\left\{\left[1-\frac{V_{A, B}}{3}(\bar{a}-\delta a)^{2}\right]^{3 / 2}-\left[1-\frac{V_{A, B}}{3} a_{0}^{2}\right]^{3 / 2}\right\} .
$$

We note that this expression is well behaved in the limit $a_{0} \rightarrow 0$, therefore in the semiclassical approach we are working on there is nothing that prevent us to safely choose the origin to be at $a_{0}=a(s=0)=0$. Thus we finally obtain

$$
-4 \pi^{2} i \int_{0}^{\bar{s}-\delta s} \frac{d s}{C(s)}\left[-3 a+a^{3} V_{A, B}\right]=\mp \frac{12 \pi^{2}}{V_{A, B}}\left\{\left[1-\frac{V_{A, B}}{3}(\bar{a}-\delta a)^{2}\right]^{3 / 2}-1\right\} .
$$

In analogy with the computation of this type of transition probabilities in the Euclidean regime, we can define a tension $T$ as the contribution to the action coming from the portion of the path in which the scalar field is not a constant in the following way

$$
2 \pi^{2} \bar{a}^{3} T=-4 \pi^{2} i \int_{\bar{s}-\delta s}^{\bar{s}+\delta s} \frac{d s}{C(s)} a^{3}\left[V(\phi)-V_{A}\right] .
$$

Then, substituting back the above equation (32) into (27) we obtain the decay rate

$$
\pm \Gamma=\mp \frac{12 \pi^{2}}{\hbar}\left\{\frac{1}{V_{B}}\left[\left(1-\frac{V_{B}}{3}(\bar{a}-\delta a)^{2}\right)^{3 / 2}-1\right]-\frac{1}{V_{A}}\left[\left(1-\frac{V_{A}}{3}(\bar{a}-\delta a)^{2}\right)^{3 / 2}-1\right]\right\}+\frac{2 \pi^{2}}{\hbar} \bar{a}^{3} T .
$$

Notice that the sign ambiguity \pm in the left-hand side is not related to the sign ambiguity $\mp$ on the right-hand side, we could choose a sign in the right hand and preserve the \pm in the left since they come from different arguments. ${ }^{1}$ This result was derived in [11] but by proposing the value $C^{2}(s)=-1$ which we note that it is inconsistent with the general system of equations (7) and (10), but nevertheless gives the correct expression for the classical action (29).

We can recover the Euclidean result for the tunnelling probability when we apply the thin wall approximation, that is when $\delta s \rightarrow 0$, in this case we have

$$
\pm \Gamma=\mp \frac{12 \pi^{2}}{\hbar}\left\{\frac{1}{V_{B}}\left[\left(1-\frac{V_{B}}{3} \bar{a}^{2}\right)^{3 / 2}-1\right]-\frac{1}{V_{A}}\left[\left(1-\frac{V_{A}}{3} \bar{a}^{2}\right)^{3 / 2}-1\right]\right\}+\frac{2 \pi^{2}}{\hbar} \bar{a}^{3} T .
$$

Looking for the points that extremize the latter expression, that is $\bar{a}$ such that $\frac{\partial \Gamma}{\partial \bar{a}}=0$, we obtain

$$
\frac{1}{\bar{a}^{2}}=\frac{V_{B}}{3}+\left(\frac{\Delta V}{3 T}+\frac{T}{4}\right)^{2}=\frac{V_{A}}{3}+\left(\frac{\Delta V}{3 T}-\frac{T}{4}\right)^{2}
$$

where $\Delta V=V_{A}-V_{B}$. Substituting back the above value of $\bar{a}$ into equation (34) and choosing the plus sign in the right hand side, we finally obtain

$$
\pm \Gamma=\frac{12 \pi^{2}}{\hbar}\left\{\frac{\left[4\left(V_{A}-V_{B}\right)^{2}+3 T^{2}\left(V_{A}+V_{B}\right)\right] \bar{a}}{12 T V_{A} V_{B}}+\frac{1}{V_{A}}-\frac{1}{V_{B}}\right\}
$$

\footnotetext{
${ }^{1}$ This argument holds for any equations involving two sign ambiguities in this work.
} 
These results coincide with the Euclidean computation for this case presented in [7]. We note that this result is valid for any nonzero values of $V_{A}$ and $V_{B}$, they could be positive or negative. For the case in which one of the potential is zero, the integral in (29) would give as a result a dependence of the form $\bar{a}^{2}$ and the following equations must be changed accordingly.

We note that the expression for the logarithm of the transition probability (36) is written in terms of two factors, namely $\bar{a}$ and $T$. However, as we have shown in (35) they are not independent, therefore, effectively the logarithm of the probability is described in this case in terms of just one degree of freedom. From (35) we can obtain a second order equation for $T$, with solutions

$$
T= \pm 2\left(\sqrt{\frac{1}{\bar{a}^{2}}-\frac{V_{A}}{3}}-\sqrt{\frac{1}{\bar{a}^{2}}-\frac{V_{B}}{3}}\right) .
$$

Then substituting it back in (34) and choosing the plus sign in the right-hand side we obtain

$$
\pm \Gamma=\frac{12 \pi^{2}}{\hbar}\left\{\frac{1}{V_{B}}\left[\left(1-\frac{V_{B}}{3} \bar{a}^{2}\right)^{3 / 2}-1\right]-\frac{1}{V_{A}}\left[\left(1-\frac{V_{A}}{3} \bar{a}^{2}\right)^{3 / 2}-1\right]+\frac{\bar{a}^{2}}{3}\left(\sqrt{1-\frac{V_{A}}{3} \bar{a}^{2}}-\sqrt{1-\frac{V_{B}}{3} \bar{a}^{2}}\right)\right\} .
$$

Therefore, we note from (37) that in the limit $\bar{a} \rightarrow 0$ the tension vanishes. Consequently, the logarithm of the transition probability (38) in this limit is well behaved and it actually goes to zero. Therefore, the transition probability goes to 1 in the limit $\bar{a} \rightarrow 0$. Thus, even if we are working in a semiclassical approach in GR we can obtain a well-defined transition probability in the ultraviolet limit, so we think our approach is worth studying despite its limited nature.

The transition probability just obtained depends on the possibility to choose a path in field space such that the scalar field depends on the parameter $s$ as in (24). This choice was useful to obtain the Euclidean results but we could also choose more general paths. For instance, we could choose the parameter $s$ as the distance in field space along the trajectories, that is we could take

$$
d s^{2}=\int_{X} G_{M N} d \Phi^{M} \Phi^{N} .
$$

By solving the resulting system we obtain that the general result for the classical action (9) takes the form

$$
\begin{aligned}
S_{0}= & \pm 2 \pi \int_{a_{0}, \phi_{B}}^{a_{m}, \phi_{A}} \frac{\left(a^{3} V(\phi)-3 a\right)^{3 / 2}}{\sqrt{\frac{3}{2 a}\left(a^{2} V(\phi)-1\right)^{2}-a^{3}\left(V^{\prime}(\phi)\right)^{2}}} \\
& \times \sqrt{a^{3} d \phi^{2}-6 a d a^{2}},
\end{aligned}
$$

where prime denotes the derivative with respect to the scalar field. We can see from the Hamiltonian constraint (20) that the last term, when taken as derivatives with respect to time, is proportional to the kinetic part of the WDW equation as

$$
\begin{aligned}
a^{3} \dot{\phi}^{2}-6 a \dot{a}^{2} & =2 N^{2}\left[\frac{\pi_{\phi}^{2}}{2 a^{3}}-\frac{\pi_{a}^{2}}{12 a}\right] \\
& =2 N^{2}\left[3 a-a^{3} V(\phi)\right],
\end{aligned}
$$

and we note from (16) that the term contributing to the transition probability is the imaginary part of the classical action. Therefore, if the integrand of equation (40) is real, then by using the last expression we note that it is possible to obtain an imaginary contribution to the action just by having the correct relations for the momenta $\pi_{A}$ and $\pi_{\phi}$, that is, such that the last expression is negative. This is possible in general because the metric in superspace is not positive definite and therefore it is possible to obtain a classical path.

\section{TRANSITIONS IN A FLAT FLRW UNIVERSE}

In this section we study the transitions in the case of a FLRW metric with zero curvature. In this case we can write the metric in cartesian coordinates as

$$
d s^{2}=-N(t) d t^{2}+a^{2}(t)\left[d x^{2}+d y^{2}+d z^{2}\right] .
$$

As usual in this case, the gravitational and scalar fields depend only on the time variable $t$. Thus the corresponding Lagrangian is given by

$$
\mathcal{L}=-\frac{3 a(t) \dot{a}(t)^{2}}{N(t)}+\frac{a^{3}(t) \dot{\phi}^{2}}{2 N(t)}-N(t) a^{3}(t) V(\phi) .
$$

The canonical momenta are the same as in Eq. (19), and the Hamiltonian constraint is

$$
H=N\left[\frac{\pi_{\phi}^{2}}{2 a^{3}}-\frac{\pi_{a}^{2}}{12 a}+a^{3} V\right] \approx 0
$$

As in the previous case we focus on the terms within brackets and proceed to identify the metric in minisuperspace and the function $f$. However, we can also factorize the Hamiltonian as follows 


$$
H=\frac{N}{a^{3}}\left[\frac{\pi_{\phi}^{2}}{2}-\frac{a^{2} \pi_{a}^{2}}{12}+a^{6} V\right] \approx 0,
$$

and then focus on the terms within brackets. This choice is analogous quantum mechanically to take a different ordering factor to obtain the WDW equation. Comparing it with the general form (1) we see that for this metric we have the coordinates $\left\{\Phi^{M}\right\}=\{a, \phi\}$ and the nonzero elements of the metric in minisuperspace are

$$
G^{a a}=-\frac{a^{2}}{6}, \quad G^{\phi \phi}=1,
$$

and the function $f$ reads

$$
f(a, \phi)=a^{6} V(\phi) .
$$

We also note that in this case the spatial volume takes the form

$$
\operatorname{Vol}(X)=\iiint d x d y d z
$$

which is finite only if we restrict ourselves to a finite interval in the variables since in this case the spatial slice is not compact and its volume might diverge. We note however, that since we are working on minisuperspace, all fields depend only on time. Therefore, we would consider a regularized version of the probability transitions by taking an spatial section that is appropriately compactified and which leads to a finite value for this term. The corresponding probability transitions will be also bounded from above and they will be finite and still might be useful to describe these transitions for the noncompact cases. In any case, the following procedure is valid without changes. ${ }^{2}$

We consider the parameter $s$ defined as before in (24), then we have using Eq. (9) for the classical actions

$$
\begin{aligned}
S_{0}\left(a_{0}, \phi_{B} ; a_{m}, \phi_{A}\right)= & -2 \operatorname{Vol}(X) \int_{0}^{s_{M}} \frac{d s}{C(s)}\left[a^{6} V(\phi)\right] \\
= & -2 \operatorname{Vol}(X)\left[\int_{0}^{\bar{s}-\delta s} \frac{d s}{C(s)}\left[a^{6} V_{B}\right]\right. \\
& +\int_{\bar{s}-\delta s}^{\bar{s}+\delta s} \frac{d s}{C(s)}\left[a^{6} V(\phi)\right] \\
& \left.+\int_{\bar{s}+\delta s}^{s_{M}} \frac{d s}{C(s)}\left[a^{6} V_{A}\right]\right], \\
S_{0}\left(a_{0}, \phi_{A} ; a_{m}, \phi_{A}\right)= & -2 \operatorname{Vol}(X) \int_{0}^{s_{M}} \frac{d s}{C(s)}\left[a^{6} V_{A}\right] .
\end{aligned}
$$

\footnotetext{
${ }^{2}$ This argument holds for any volume term that does not come from a compact spatial slice for the following sections of this article.
}

Substituting these expressions into Eq. (15) we obtain the decay rate

$$
\begin{aligned}
\pm \Gamma= & -\frac{2 \operatorname{Vol}(X) i}{\hbar} \int_{0}^{\bar{s}-\delta s} \frac{d s}{C(s)}\left[a^{6} V_{B}\right] \\
& +\frac{2 \operatorname{Vol}(X) i}{\hbar} \int_{0}^{\bar{s}-\delta s} \frac{d s}{C(s)}\left[a^{6} V_{A}\right] \\
& -\frac{2 \operatorname{Vol}(X) i}{\hbar} \int_{\bar{s}-\delta s}^{\bar{s}+\delta s} \frac{d s}{C(s)} a^{6}\left[V(\phi)-V_{A}\right] .
\end{aligned}
$$

For the case when the scalar field is constant, we use Eqs. (7) and (10) to compute the first two integrals of Eq. (51) for the flat FLRW metric. The general solutions (11) and (12) take in this case the form

$$
\begin{aligned}
C^{2}(s) & =12 \operatorname{Vol}^{2}(X) a^{6} V_{A, B}, \\
\frac{d a}{d s} & =\frac{1}{\operatorname{Vol}(X)} \frac{a}{6} .
\end{aligned}
$$

In terms of the variable $a(s)$ we obtain

$-2 \operatorname{Vol}(X) \int \frac{d s}{C(s)}\left[a^{6} V_{A, B}\right]= \pm 2 \operatorname{Vol}(X) \sqrt{\frac{V_{A, B}}{3}} a^{3}$.

We note that again we can safely choose the origin to be at $a_{0}=0$, then we get

$$
\begin{aligned}
- & 2 \operatorname{Vol}(X) \int_{0}^{\bar{s}-\delta s} \frac{d s}{C(s)}\left[a^{6} V_{A, B}\right] \\
& = \pm 2 \operatorname{Vol}(X) \sqrt{\frac{V_{A, B}}{3}}(\bar{a}-\delta a)^{3} .
\end{aligned}
$$

In this case we can also define a tension term $T$ as the contribution coming from the portion of the path in which the scalar field is not a constant as follows

$\operatorname{Vol}(X) \bar{a}^{6} T=-2 \operatorname{Vol}(X) i \int_{\bar{s}-\delta s}^{\bar{s}+\delta s} \frac{d s}{C(s)} a^{6}\left[V(\phi)-V_{A}\right]$

Then, substituting the previous expression into (51) and considering the thin wall limit of interest, that is $\delta s \rightarrow 0$, we obtain

$\pm \Gamma= \pm \frac{2 i \operatorname{Vol}(X)}{\sqrt{3} \hbar}\left(\sqrt{V_{B}}-\sqrt{V_{A}}\right) \bar{a}^{3}+\frac{\operatorname{Vol}(X)}{\hbar} \bar{a}^{6} T$.

Looking for the points that extremize the latter expression, that is $\frac{\partial \Gamma}{\partial \bar{a}}=0$, we obtain two possible solutions

$$
\bar{a}^{3}=\left\{\begin{array}{l}
0, \\
\mp \frac{i}{\sqrt{3} T}\left(\sqrt{V_{B}}-\sqrt{V_{A}}\right) .
\end{array}\right.
$$


The first option of (57) implies the vanishing of expression (56), so we focus on the second line. Since $\bar{a}$ should be real, we find that in order to have an extremum for $\Gamma$ different from zero, we must choose both $V_{A}$ and $V_{B}$ to be negative, in which case we obtain

$$
\bar{a}^{3}= \pm \frac{1}{\sqrt{3} T}\left(\sqrt{\left|V_{B}\right|}-\sqrt{\left|V_{A}\right|}\right)
$$

and substituting back into (56) we finally obtain

$$
\pm 2 \operatorname{Re}[\Gamma]=-\frac{2 \operatorname{Vol}(X)}{3 \hbar T}\left(\sqrt{\left|V_{B}\right|}-\sqrt{\left|V_{A}\right|}\right)^{2}
$$

For non-negative values of $V_{A, B}$ we obtain in general

$$
\begin{aligned}
\pm 2 \operatorname{Re}[\Gamma]= & \mp \frac{4 \operatorname{Vol}(X)}{\sqrt{3} \hbar} \operatorname{Im}\left[\left(\sqrt{V_{B}}-\sqrt{V_{A}}\right)\right] \bar{a}^{3} \\
& +\frac{2 \operatorname{Vol}(X)}{\hbar} \bar{a}^{6} T
\end{aligned}
$$

but without an extremum. In particular, notice that for a transition between two dS spaces values, the only term contributing is the tension term.

We note also that in any of the possible cases, that is, if we regard $T$ as an independent term or related to $\bar{a}$ as in (58) whenever it is possible, the logarithm of the transition probability (60) is well behaved in the ultraviolet limit $\bar{a} \rightarrow 0$ giving once again a vanishing value for $\Gamma$.

\section{TRANSITIONS IN KANTOWSKI-SACHS}

Now that we have studied the simplest cases of isotropic universes, let us move on to the study of anisotropic metrics. Let us start with the Kantowski-Sachs metric. Using the parametrization of this metric used by Misner in [23] we have

$$
\begin{aligned}
d s^{2}= & -N^{2}(t) d t^{2}+e^{2 \sqrt{3} \beta(t)} d r^{2} \\
& +e^{-2 \sqrt{3}(\beta(t)+\Omega(t))}\left[d \theta^{2}+\sin ^{2}(\theta) d \psi^{2}\right],
\end{aligned}
$$

with $0 \leq \theta \leq \pi$ and $0 \leq \psi \leq 2 \pi$. Let us define for simplicity the functions $\gamma(t)=e^{\sqrt{3} \beta(t)}$ and $\sigma(t)=e^{-\sqrt{3} \Omega(t)}$, then the metric is written as

$$
\begin{aligned}
d s^{2}= & -N^{2}(t) d t^{2}+\gamma^{2}(t) d r^{2} \\
& +\frac{\sigma^{2}(t)}{\gamma^{2}(t)}\left[d \theta^{2}+\sin ^{2} \theta d \psi^{2}\right] .
\end{aligned}
$$

Considering again gravity coupling to a scalar field depending only on the time variable $\phi(t)$ with potential $V(\phi)$ and using natural units, the Lagrangian is

$$
\begin{aligned}
\mathcal{L}= & \frac{\sigma^{2}(t)}{\gamma^{3}(t) N(t)} \dot{\gamma}^{2}-\frac{\dot{\sigma}^{2}}{\gamma(t) N(t)}+N(t) \gamma(t) \\
& +\left[\frac{\dot{\phi}^{2}}{2 N(t)}-N(t) V(\phi)\right] \frac{\sigma^{2}(t)}{\gamma(t)}
\end{aligned}
$$

The canonical momenta are

$\pi_{N}=0, \quad \pi_{\gamma}=\frac{2 \sigma^{2}}{N \gamma^{3}} \dot{\gamma}, \quad \pi_{\sigma}=-\frac{2}{N \gamma} \dot{\sigma}, \quad \pi_{\phi}=\frac{\sigma^{2}}{N \gamma} \dot{\phi}$

and the Hamiltonian constraint turns out to be

$H=N\left[\frac{\gamma^{3}}{4 \sigma^{2}} \pi_{\gamma}^{2}-\frac{\gamma}{4} \pi_{\sigma}^{2}+\frac{\gamma}{2 \sigma^{2}} \pi_{\phi}^{2}+\frac{\sigma^{2}}{\gamma} V(\phi)-\gamma\right] \approx 0$.

Again, we can focus only on the term inside brackets, and comparing it with the general form (1) we see that the set of coordinates is $\left\{\Phi^{M}\right\}=\{\gamma, \sigma, \phi\}$, the nonvanishing element on the metric in minisuperspace are

$$
G^{\gamma \gamma}=\frac{\gamma^{3}}{2 \sigma^{2}}, \quad G^{\sigma \sigma}=-\frac{\gamma}{2}, \quad G^{\phi \phi}=\frac{\gamma}{\sigma^{2}},
$$

and the function $f$ is given by

$$
f(\gamma, \sigma, \phi)=\frac{\sigma^{2}}{\gamma} V(\phi)-\gamma
$$

We also have in this case that the volume of $X$ reads

$\operatorname{Vol}(X)=\int_{r} \int_{\theta=0}^{\pi} \int_{\psi=0}^{2 \pi} \sin \theta d r d \theta d \psi=4 \pi \int d r$

which is finite only if we restrict ourselves to a finite interval on $r$, since in this case again the spatial slice is not compact.

We choose the parameter $s$ as before in Eq. (24), therefore by using (9) we obtain in this case that the classical actions are given by

$$
\begin{aligned}
S_{0}\left(\gamma_{0}, \sigma_{0}, \phi_{B} ; \gamma_{m}, \sigma_{m}, \phi_{A}\right) \\
=-2 \operatorname{Vol}(X)\left\{\int_{0}^{\bar{s}-\delta s} \frac{d s}{C(s)}\left[\frac{\sigma^{2}}{\gamma} V_{B}-\gamma\right]\right. \\
+\int_{\bar{s}-\delta s}^{\bar{s}+\delta s} \frac{d s}{C(s)}\left[\frac{\sigma^{2}}{\gamma} V(\phi)-\gamma\right] \\
\left.+\int_{\bar{s}+\delta s}^{s_{M}} \frac{d s}{C(s)}\left[\frac{\sigma^{2}}{\gamma} V_{A}-\gamma\right]\right\},
\end{aligned}
$$

$S_{0}\left(\gamma_{0}, \sigma_{0}, \phi_{A} ; \gamma_{m}, \sigma_{m}, \phi_{A}\right)=-2 \operatorname{Vol}(X) \int_{0}^{s_{M}} \frac{d s}{C(s)}\left[\frac{\sigma^{2}}{\gamma} V_{A}-\gamma\right]$, 
and then substituting into (15) we obtain the corresponding decay rate

$$
\begin{aligned}
\pm \Gamma= & -\frac{2 \operatorname{Vol}(X) i}{\hbar} \int_{0}^{\bar{s}-\delta s} \frac{d s}{C(s)}\left[\frac{\sigma^{2}}{\gamma} V_{B}-\gamma\right] \\
& +\frac{2 \operatorname{Vol}(X) i}{\hbar} \int_{0}^{\bar{s}-\delta s} \frac{d s}{C(s)}\left[\frac{\sigma^{2}}{\gamma} V_{A}-\gamma\right] \\
& -\frac{2 \operatorname{Vol}(X) i}{\hbar} \int_{\bar{s}-\delta s}^{\bar{s}+\delta s} \frac{d s}{C(s)} \frac{\sigma^{2}}{\gamma}\left[V(\phi)-V_{A}\right] .
\end{aligned}
$$

To compute the first two integrals we again study the system of equations corresponding to this metric in the case in which the scalar field is constant. The general solutions (11) and (12) in this case read

$$
\begin{aligned}
C^{2}(s) & =\operatorname{Vol}^{2}(X)\left(\frac{\gamma^{2}}{\sigma^{2}}+3 V_{A, B}\right), \\
\frac{d \gamma}{d s} & =\frac{1}{\operatorname{Vol}(X)} \frac{\gamma\left(\frac{\gamma^{2}}{\sigma^{2}}+V_{A, B}\right)}{\frac{\gamma^{2}}{\sigma^{2}}+3 V_{A, B}}, \\
\frac{d \sigma}{d s} & =\frac{1}{\operatorname{Vol}(X)} \frac{2 \sigma V_{A, B}}{\frac{\gamma^{2}}{\sigma^{2}}+3 V_{A, B}} .
\end{aligned}
$$

Now let us assume that neither $V_{A}$ nor $V_{B}$ are zero, then the relations (13) in this case give us

$$
\frac{1}{\gamma\left(\frac{\gamma^{2}}{\sigma^{2}}+V_{A, B}\right)} d \gamma=\frac{1}{2 \sigma V_{A, B}} d \sigma,
$$

which can be written as the differential equation

$$
2 \sigma V_{A, B} d \gamma-\gamma\left(\frac{\gamma^{2}}{\sigma^{2}}+V_{A, B}\right) d \sigma=0
$$

This last expression leads to the relation

$$
\gamma^{2}=\frac{\sigma^{2} V_{A, B}}{1-c \sigma}
$$

where $c$ is an integration constant. We note that by definition $\gamma$ and $\sigma$ are positive functions, therefore the constant $c$ has to fulfill the condition

$$
\frac{V_{A, B}}{1-c \sigma}>0
$$

which depends on the value of $V_{A, B}$ and the possible values that $\sigma$ can have. Having these two fields related, we can express the first two integrals in (71) in terms of $\sigma$ only. Moreover, changing the integration variable as $d s=$ $\left(\frac{d \sigma}{d s}\right)^{-1} d \sigma$ we can perform the integral to obtain

$$
\begin{gathered}
-2 \operatorname{Vol}(X) \int \frac{d s}{C(s)}\left[\frac{\sigma^{2}}{\gamma} V_{A, B}-\gamma\right] \\
= \pm \operatorname{Vol}(X) c_{A, B} F_{K S}\left[c_{A, B}, \sigma\right],
\end{gathered}
$$

where we have defined the function

$$
\begin{aligned}
F_{K S}[c, x]= & \int \frac{\sqrt{4-3 c x}}{1-c x} x d x \\
= & -\frac{2}{9 c^{2}}[\sqrt{4-3 c x}(5+3 c x) \\
& -9 \operatorname{arctanh}(\sqrt{4-3 c x})] .
\end{aligned}
$$

In analogy with the FLRW metric (32), we can also define now a tension term as

$\operatorname{Vol}(X) \frac{\bar{\sigma}^{2}}{\bar{\gamma}} T=-2 \operatorname{Vol}(X) i \int_{\bar{s}-\delta s}^{\bar{s}+\delta s} \frac{d s}{C(s)} \frac{\sigma^{2}}{\gamma}\left[V(\phi)-V_{A}\right]$,

but as we have shown the minisuperspace coordinates $\gamma$ and $\sigma$ can be related. In fact, using the relation obtained in the case in which the scalar field is a constant (75) and the way in which the parameter $s$ was defined in (24), we have

$$
\gamma= \begin{cases}\sigma \sqrt{\frac{V_{B}}{1-c_{B} \sigma}}, & 0<s<\bar{s}-\delta s, \\ \gamma(\sigma), & \bar{s}-\delta s<s<\bar{s}+\delta s, \\ \sigma \sqrt{\frac{V_{A}}{1-c_{A A} \sigma}}, & \bar{s}+\delta s<s<s_{M},\end{cases}
$$

where $c_{A A}$ is a constant that satisfies the condition (76) but it is in general different from $c_{A}$ in (77) since that constant emerges on a different path. In the last expression $\gamma(\sigma)$ in the middle interval can be found in principle by solving the system of equations for the case in which the scalar field can vary. However, we note that in the thin wall limit in which we are interested to work with, $\delta s \rightarrow 0$, the latter simplifies to

$$
\gamma= \begin{cases}\sigma \sqrt{\frac{V_{B}}{1-c_{B} \sigma}}, & 0<s<\bar{s}-\delta s, \\ \sigma \sqrt{\frac{V_{A}}{1-c_{A A} \sigma}}, & \bar{s}+\delta s<s<s_{M},\end{cases}
$$

and we can impose continuity on $\gamma$ to obtain

$$
\begin{aligned}
\bar{\gamma} & =\bar{\sigma} \sqrt{\frac{V_{B}}{1-c_{B} \bar{\sigma}}}=\bar{\sigma} \sqrt{\frac{V_{A}}{1-c_{A A} \bar{\sigma}}} \\
& =\frac{V_{B}-V_{A}}{\sqrt{\left(c_{B}-c_{A A}\right)\left(c_{B} V_{A}-c_{A A} V_{B}\right)}},
\end{aligned}
$$

and 


$$
\bar{\sigma}=\frac{V_{A}-V_{B}}{c_{B} V_{A}-c_{A A} V_{B}} .
$$

Substituting the above results in (79) and gathering it and the result (77) in (71) we then obtain in the thin wall limit

$$
\begin{aligned}
\pm \Gamma= & \pm \frac{\operatorname{Vol}(X) i}{\hbar}\left[\left.c_{B} F_{K S}\left[c_{B}, \sigma\right]\right|_{\sigma_{0}} ^{\bar{\sigma}}-\left.c_{A} F_{K S}\left[c_{A}, \sigma\right]\right|_{\sigma_{0}} ^{\bar{\sigma}}\right] \\
& +\frac{\operatorname{Vol}(X)}{\hbar} \frac{\bar{\sigma} T}{\sqrt{V_{B}}} \sqrt{1-c_{B} \bar{\sigma}}
\end{aligned}
$$

Therefore, in this limit we obtain for $\Gamma$ an expression that only depends on two variables, $\bar{\sigma}$ and $T$, in complete analogy to the FLRW cases. Note that in this case we can also safely choose $\sigma_{0}=0$ since the above expression is well behaved in that limit. If we want to find an extremum of this expression, that is $\bar{\sigma}$ such that $\frac{\partial \gamma}{\partial \bar{\sigma}}=0$, we obtain that the following equation has to be hold

$\frac{2-3 c_{B} \bar{\sigma}}{2 \sqrt{V_{B}\left(1-c_{B} \bar{\sigma}\right)}} T=\mp i \bar{\sigma}\left[\frac{c_{B} \sqrt{4-3 c_{B} \bar{\sigma}}}{1-c_{B} \bar{\sigma}}-\frac{c_{A} \sqrt{4-3 c_{A} \bar{\sigma}}}{1-c_{A} \bar{\sigma}}\right]$.

We note in particular that for two positive values of the potentials, the last equation have as only solution $T=0$ which is not of interest to us, and therefore, there is not a minimum with a nonzero tension in that case.

Since we expect the constants to be real numbers, we finally obtain

$$
\begin{aligned}
\pm 2 \operatorname{Re}[\Gamma]= & \frac{2 \operatorname{Vol}(X)}{\hbar}\left\{\mp \left[\left.c_{B} \operatorname{Im}\left[F_{K S}\left[c_{B}, \sigma\right]\right]\right|_{\sigma_{0}} ^{\bar{\sigma}}\right.\right. \\
& \left.-\left.c_{A} \operatorname{Im}\left[F_{K S}\left[c_{A}, \sigma\right]\right]\right|_{\sigma_{0}} ^{\bar{\sigma}}\right] \\
& \left.+\frac{\bar{\sigma} T}{\sqrt{V_{B}}} \sqrt{1-c_{B} \bar{\sigma}}\right\} .
\end{aligned}
$$

Note that this result and the latter equations are valid for any values of the potentials different from zero. The only difference between positive or negative potentials are the choices of the integration constants so the condition (76) holds in each particular case. Once again we find that if we regard $T$ as an independent parameter for positive values of the potentials, or related to $\bar{\sigma}$ as in (85), the limit $\bar{\sigma} \rightarrow$ $\sigma_{0}=0$ is well behaved and it is a regular function in this limit. Equation (85) actually gives $T \rightarrow 0$ in this limit, and in general it yields a vanishing value for $\Gamma$, allowing us to study the ultraviolet limit.

For the case in which the potential is zero, the general solution (11) and (12) gives

$$
\begin{aligned}
C^{2}(s) & =\operatorname{Vol}^{2}(X) \frac{\gamma^{2}}{\bar{\sigma}^{2}}, \\
\frac{d \gamma}{d s} & =\frac{\gamma}{\operatorname{Vol}(X)}, \\
\frac{d \sigma}{d s} & =0, \quad \therefore \sigma=\bar{\sigma},
\end{aligned}
$$

then we obtain for this case

$$
-\left.2 \operatorname{Vol}(X) \int \frac{d s}{C(s)}\left[\frac{\sigma^{2}}{\gamma} V-\gamma\right]\right|_{V=0}= \pm 2 \operatorname{Vol}(X) \bar{\sigma} \ln \gamma
$$

Let us choose in the first instance $V_{A}=0$, then $V_{B}<0$, in this case the path in which the system tunnels from one minimum to the other, $\sigma$ starts with one value and evolves until it reaches a constant $\bar{\sigma}$ and then it remains at that value. For this case we obtain

$$
\begin{aligned}
\pm \Gamma= & \pm \frac{\operatorname{Vol}(X) i}{\hbar}\left[\left.c_{B} F_{K S}\left[c_{B}, \sigma\right]\right|_{\sigma_{0}} ^{\bar{\sigma}}-2 \bar{\sigma} \ln \gamma \mid \begin{array}{l}
\bar{\sigma} \sqrt{\frac{V_{B}}{1-c_{B} \bar{\sigma}}} \\
\sigma_{0} \sqrt{\frac{V_{B}}{1-c_{B} \sigma_{0}}}
\end{array}\right] \\
& +\frac{\operatorname{Vol}(X)}{\hbar} \frac{\bar{\sigma} T}{\sqrt{V_{B}}} \sqrt{1-c_{B} \bar{\sigma}}
\end{aligned}
$$

from this we note that we cannot choose $\sigma_{0}=0$ since there is a divergence in the logarithm. The condition to have an extremum leads to the equation

$$
\frac{2-3 c_{\sigma} \overline{\bar{\sigma}}}{2 \sqrt{V_{B}\left(1-c_{\sigma} \bar{\sigma}\right)}} T=\mp i\left\{\frac{\sqrt{4-3 c_{\sigma} \bar{\sigma}}}{1-c_{\sigma} \bar{\sigma}} c_{\sigma} \bar{\sigma}-2\left[\ln \left(\bar{\sigma} \sqrt{\frac{V_{B}}{1-c_{\sigma} \bar{\sigma}}}\right)-\ln \left(\sigma_{0} \sqrt{\frac{V_{B}}{1-c_{\sigma} \sigma_{0}}}\right)+1-\frac{c_{\sigma} \bar{\sigma}}{2\left(1-c_{\sigma} \bar{\sigma}\right)}\right]\right\}
$$

In the other possible case we choose $V_{B}=0$, therefore $V_{A}>0$. In this case everything is expressed in terms of $\gamma$ instead of $\sigma$, then we have

$$
\bar{\sigma}=\frac{\bar{\gamma}}{2 V_{A}}\left[\sqrt{c_{A}^{2} \bar{\gamma}^{2}+4 V_{A}}-c_{A} \bar{\gamma}\right]
$$

and consequently 


$$
\begin{aligned}
\pm \Gamma= & \pm \frac{\operatorname{Vol}(X) i}{\hbar}\left\{\left.\frac{\bar{\gamma}}{V_{A}}\left[\sqrt{c_{A}^{2} \bar{\gamma}^{2}+4 V_{A}}-c_{A} \bar{\gamma}\right] \ln \gamma\right|_{\gamma_{0}} ^{\bar{\gamma}}-\left.c_{A} F_{K S}\left[c_{A}, \frac{\gamma}{2 V_{A}}\left(\sqrt{c_{A}^{2} \gamma^{2}+4 V_{A}}-c_{A} \gamma\right)\right]\right|_{\gamma_{0}} ^{\bar{\gamma}}\right\} \\
& +\frac{\operatorname{Vol}(X)}{\hbar} \frac{\bar{\gamma} T}{4 V_{A}^{2}}\left(\sqrt{c_{A}^{2} \bar{\gamma}^{2}+4 V_{A}}-c_{A} \bar{\gamma}\right)^{2},
\end{aligned}
$$

in this case we also note that $\gamma_{0}=0$ is not possible because it causes a divergence. The condition for this expression to have an extremum is fulfilled if the following equation holds

$$
\begin{aligned}
\frac{T}{4 V_{A}^{2}}\left[6 c_{A}^{2} \bar{\gamma}^{2}+4 V_{A}-\frac{2 c_{A} \bar{\gamma}}{\bar{d}}\left(3 c_{A}^{2} \bar{\gamma}^{2}+8 V_{A}\right)\right]= & \mp i\left[-\frac{c_{A}}{V_{A}}\left(\frac{c_{A}^{2} \bar{\gamma}^{2}+2 V_{A}}{\bar{d}}-c_{A} \bar{\gamma}\right) \frac{\sqrt{4-\frac{3 c_{A}}{2 V_{A}} \bar{\gamma}}}{1-\frac{c_{A}}{2 V_{A}} \bar{\gamma}}+\frac{\bar{b}}{V_{A}}\right. \\
& \left.+\frac{2}{V_{A}}\left(\frac{c_{A}^{2} \bar{\gamma}^{2}+2 V_{A}}{\bar{d}}-c_{A} \bar{\gamma}\right)\left(\ln \bar{\gamma}-\ln \gamma_{0}\right)\right],
\end{aligned}
$$

where

$$
d=\sqrt{c_{A}^{2} \bar{\gamma}^{2}+4 V_{A}}, \quad \bar{b}=\bar{d}-c_{A} \bar{\gamma} .
$$

As we mentioned in the final part of Sec. III, we could also used the distance along the trajectories in field space as the parameter $s$ defined in (39). In this case, after solving the system of equations we obtain for the classical action the general expression

$$
S_{0}= \pm 2 \sqrt{\operatorname{Vol}(X)} \int_{\gamma_{0}, \sigma_{0}, \phi_{B}}^{\gamma_{m}, \sigma_{m}, \phi_{A}} \frac{\sigma^{2}\left(V(\phi)-\frac{\gamma^{2}}{\sigma^{2}}\right)^{3 / 2}}{\gamma \sqrt{\left(3 V(\phi)+\frac{\gamma^{2}}{\sigma^{2}}\right)\left(V(\phi)-\frac{\gamma^{2}}{\sigma^{2}}\right)-2\left(V^{\prime}(\phi)\right)^{2}}} d s
$$

where

$$
d s=\sqrt{\frac{2 \sigma^{2}}{\gamma^{3}} d \gamma^{2}-\frac{2}{\gamma} d \sigma^{2}+\frac{\sigma^{2}}{\gamma} d \phi^{2}}
$$

and using the Hamiltonian constraint (65) we can write the $d s$ term as

$$
\begin{aligned}
\frac{2 \sigma^{2}}{\gamma^{3}} \dot{\gamma}^{2}-\frac{2}{\gamma} \dot{\sigma}^{2}+\frac{\sigma^{2}}{\gamma} \dot{\phi}^{2} & =2 N^{2}\left[\frac{\gamma^{3}}{4 \sigma^{2}} \pi_{\gamma}^{2}-\frac{\gamma}{4} \pi_{\sigma}^{2}+\frac{\gamma}{2 \sigma^{2}} \pi_{\phi}^{2}\right] \\
& =2 N^{2}\left[\gamma-\frac{\sigma^{2}}{\gamma} V(\phi)\right]
\end{aligned}
$$

Therefore, if the integrand in (95) is real, we can have contributions for the transition probability just by having a negative kinetic term in the WDW equation. Again, this is possible because the metric in superspace is not positive definite, allowing again for a classical path. We note that in this case, since we have three terms, we have more possibilities to realize these paths. Since we have only one negative term coming from the momenta $\pi_{\sigma}$, it may seem from the first equality that we could have transitions even if the scalar field is not considered. However, if we remove the scalar field from (95) we obtain

$$
\left.S_{0}\right|_{\phi=0, V=0}=\mp 2 \sqrt{\operatorname{Vol}(X)} \int_{\gamma_{0}, \sigma_{0}}^{\gamma_{m}, \sigma_{m}} \sigma \sqrt{\frac{2 \sigma^{2}}{\gamma^{3}} d \gamma^{2}-\frac{2}{\gamma} d \sigma^{2}},
$$

and from the second equality in (97) we note that the last term cannot be negative, since it depends only on $\gamma$ in this case. Therefore, if we remove the scalar field, we have no contributions for the transition probability.

\section{TRANSITIONS IN BIANCHI III}

Another important anisotropic metric is the Bianchi III metric. In this section we study probability transitions for this anisotropic kind of models. The metric is of the form [24]

$$
\begin{aligned}
d s^{2}= & -N^{2}(t) d t^{2}+A^{2}(t) d x^{2}+B^{2}(t) e^{-2 \alpha x} d y^{2} \\
& +C^{2}(t) d z^{2}
\end{aligned}
$$

where $\alpha \neq 0$ is a constant. We note that if we take $A(t)=$ $B(t)=C(t)$ and $\alpha=0$ then we recover the FLRW flat metric (42). Considering a scalar field coupled to gravity with the metric (99) we obtain the Lagrangian 


$$
\begin{aligned}
\mathcal{L}= & -\frac{1}{N(t)}(\dot{A}(t) \dot{B}(t) C(t)+\dot{A}(t) \dot{C}(t) B(t)+\dot{B}(t) \dot{C}(t) A(t)) \\
& -\frac{\alpha^{2}}{A(t)} N(t) B(t) C(t)+\frac{\dot{\phi}^{2}}{2 N(t)} A(t) B(t) C(t) \\
& -V(\phi) N(t) A(t) B(t) C(t) .
\end{aligned}
$$

The canonical momenta are

$$
\begin{aligned}
& \pi_{N}=0, \quad \pi_{A}=-\frac{1}{N}(\dot{B} C+\dot{C} B), \quad \pi_{B}=-\frac{1}{N}(\dot{A} C+\dot{C} A), \\
& \pi_{C}=-\frac{1}{N}(\dot{A} B+\dot{B} A), \quad \pi_{\phi}=\frac{\dot{\phi}}{N} A B C
\end{aligned}
$$

and the Hamiltonian takes the form

$$
\begin{aligned}
H= & N\left[\frac{A}{4 B C} \pi_{A}^{2}+\frac{B}{4 A C} \pi_{B}^{2}+\frac{C}{4 A B} \pi_{C}^{2}\right. \\
& -\frac{1}{2 C} \pi_{A} \pi_{B}-\frac{1}{2 B} \pi_{A} \pi_{C}-\frac{1}{2 A} \pi_{B} \pi_{C} \\
& \left.+\frac{\pi_{\phi}^{2}}{2 A B C}+\frac{\alpha^{2} B C}{A}+V(\phi) A B C\right] .
\end{aligned}
$$

However, as we did with the FLRW flat metric, we find it more useful to factorize the Hamiltonian as follows

$$
\begin{aligned}
H= & \frac{N}{A B C}\left\{\frac{A^{2}}{4} \pi_{A}^{2}-\frac{A B}{2} \pi_{A} \pi_{B}-\frac{A C}{2} \pi_{A} \pi_{C}\right. \\
& +\frac{B^{2}}{4} \pi_{B}^{2}-\frac{B C}{2} \pi_{B} \pi_{C}+\frac{C^{2}}{4} \pi_{C}^{2}+\frac{1}{2} \pi_{\phi}^{2} \\
& \left.+B^{2} C^{2} \alpha^{2}+A^{2} B^{2} C^{2} V(\phi)\right\},
\end{aligned}
$$

and then focus on the terms within the brackets. Comparing with the general form (1) we see that the coordinates are $\left\{\Phi^{M}\right\}=\{A, B, C, \phi\}$. Moreover the metric in the minisuperspace is written as

$$
\left(G^{M N}\right)=\frac{1}{2}\left(\begin{array}{cccc}
A^{2} & -A B & -A C & 0 \\
-A B & B^{2} & -B C & 0 \\
-A C & -B C & C^{2} & 0 \\
0 & 0 & 0 & 2
\end{array}\right)
$$

and the function $f$ reads

$$
f(A, B, C, \phi)=B^{2} C^{2}\left(\alpha^{2}+A^{2} V(\phi)\right) .
$$

We also note that in this case the volume of $X$ is given by

$$
\operatorname{Vol}(X)=\iiint e^{-\alpha x} d x d y x d z
$$

which again is finite only if we restrict to a finite interval in $y$ and $z$. This happens because the spatial slice is not compact as was the case for the flat FLRW and KantowskiSachs metrics.

Take the parameter $s$ as in (24) we obtain the classical actions

$$
\begin{aligned}
S_{0}\left(A_{0}, B_{0}, C_{0}, \phi_{B} ; A_{m}, B_{m}, C_{m}, \phi_{A}\right)= & -2 \operatorname{Vol}(X)\left[\int_{0}^{\bar{s}-\delta s} \frac{d s}{C(s)}\left[B^{2} C^{2} \alpha^{2}+A^{2} B^{2} C^{2} V_{B}\right]\right. \\
& \left.+\int_{\bar{s}-\delta s}^{\bar{s}+\delta s} \frac{d s}{C(s)}\left[B^{2} C^{2} \alpha^{2}+A^{2} B^{2} C^{2} V(\phi)\right] \int_{\bar{s}+\delta s}^{s_{M}} \frac{d s}{C(s)}\left[B^{2} C^{2} \alpha^{2}+A^{2} B^{2} C^{2} V_{A}\right]\right], \\
S_{0}\left(A_{0}, B_{0}, C_{0}, \phi_{A} ; A_{m}, B_{m}, C_{m}, \phi_{A}\right)= & -2 \operatorname{Vol}(X) \int_{0}^{s_{M}} \frac{d s}{C(s)}\left[B^{2} C^{2} \alpha^{2}+A^{2} B^{2} C^{2} V_{A}\right],
\end{aligned}
$$

and therefore

$$
\begin{aligned}
\pm \Gamma= & -\frac{2 \operatorname{Vol}(X) i}{\hbar} \int_{0}^{\bar{s}-\delta s} \frac{d s}{C(s)} B^{2} C^{2}\left[\alpha^{2}+A^{2} V_{B}\right]+\frac{2 \operatorname{Vol}(X) i}{\hbar} \int_{0}^{\bar{s}-\delta s} \frac{d s}{C(s)} B^{2} C^{2}\left[\alpha^{2}+A^{2} V_{A}\right] \\
& -\frac{2 \operatorname{Vol}(X) i}{\hbar} \int_{\bar{s}-\delta s}^{\bar{s}+\delta s} \frac{d s}{C(s)} A^{2} B^{2} C^{2}\left[V(\phi)-V_{A}\right] .
\end{aligned}
$$


Using the same procedure as in the previous examples, we have for the case in which the scalar field is constant and the potential is nonzero, the solution

$$
\begin{aligned}
C^{2}(s) & =4 \operatorname{Vol}^{2}(X) A^{2} B^{2} C^{2} V_{A, B} \frac{4 \alpha^{2}+3 A^{2} V_{A, B}}{\alpha^{2}+A^{2} V_{A, B}}, \\
\frac{d A}{d s} & =\frac{1}{\operatorname{Vol}(X)} \frac{1}{2 A V_{A, B}} \frac{\left(2 \alpha^{2}+A^{2} V_{A, B}\right)\left(\alpha^{2}+A^{2} V_{A, B}\right)}{4 \alpha^{2}+3 A^{2} V_{A, B}}, \\
\frac{d B}{d s} & =\frac{1}{\operatorname{Vol}(X)} \frac{B}{2} \frac{\alpha^{2}+A^{2} V_{A, B}}{4 \alpha^{2}+3 A^{2} V_{A, B}}, \\
\frac{d C}{d s} & =\frac{1}{\operatorname{Vol}(X)} \frac{C}{2} \frac{\alpha^{2}+A^{2} V_{A, B}}{4 \alpha^{2}+3 A^{2} V_{A, B}} .
\end{aligned}
$$

Moreover the coordinates are related as

$$
\frac{1}{C} \frac{d C}{d s}=\frac{1}{B} \frac{d B}{d s}=\frac{A V_{A, B}}{2 \alpha^{2}+A^{2} V_{A, B}} \frac{d A}{d s},
$$

from where we obtain

$$
B=b_{0} C=c_{0} \sqrt{\frac{2 \alpha^{2}}{V_{A, B}}+A^{2}}
$$

where $b_{0}$ and $c_{0}$ are integration constants. Since they are only multiplicative constants, they can be absorbed by redefinitions on the spatial variables, therefore, for the two first integrals in (109) we have respectively

$$
B=C=\sqrt{\frac{2 \alpha^{2}}{V_{A, B}}+A^{2}} .
$$

Notice that if we take $\alpha=0$, the latter equation tells us that $A=B=C$, that is, in the semiclassical approach, the only condition to recover the flat FLRW metric is $\alpha=0$. With these relations we can express the two first integrals in (109) in terms of $A$ only and then by changing the integration variable as $d s=\left(\frac{d A}{d s}\right)^{-1} d A$ we can perform the remaining integral to obtain

$$
-2 \operatorname{Vol}(X) \int \frac{d s}{C(s)} B^{2} C^{2}\left[\alpha^{2}+A^{2} V_{A, B}\right]= \pm 2 \operatorname{Vol}(X) \sqrt{V_{A, B}} F_{\mathrm{III}}\left[V_{A, B}, A\right]
$$

where we have defined the function

$$
\begin{aligned}
F_{\text {III }}[V, x]= & \int \sqrt{\left(3 x^{2}+\frac{4 \alpha^{2}}{V}\right)\left(x^{2}+\frac{\alpha^{2}}{V}\right)} d x \\
= & \frac{1}{9 \sqrt{\frac{4 \alpha^{4}}{V^{2}}+\frac{7 \alpha^{2}}{V} x^{2}+3 x^{4}}}\left\{\frac{12 \alpha^{4}}{V^{2}} x+\frac{21 \alpha^{2}}{V} x^{3}+9 x^{5}\right. \\
& \left.+\frac{2 \alpha^{3} i}{V^{5 / 2}} \sqrt{x^{2} V+\alpha^{2}} \sqrt{3 V x^{2}+4 \alpha^{2}}\left[F\left(i \arcsin h\left(\sqrt{\frac{V x}{\alpha^{2}}}\right), \frac{3}{4}\right)-7 E\left(i \arcsin h\left(\sqrt{\frac{V x}{\alpha^{2}}}\right), \frac{3}{4}\right)\right]\right\} .
\end{aligned}
$$

Here $F(x, m)$ and $E(x, m)$ are the elliptic integrals of first and second kind respectively. Note that if we choose $\alpha=0$ the integral in the last expression simplifies and (114) takes the same form as in the flat FLRW case (53). We can also define a tension term as

$$
\operatorname{Vol}(X) \bar{A}^{2} \bar{B}^{2} \bar{C}^{2} T=-2 \operatorname{Vol}(X) i \int_{\bar{s}-\delta s}^{\bar{s}+\delta s} \frac{d s}{C(s)} A^{2} B^{2} C^{2}\left[V(\phi)-V_{A}\right]
$$

With the solutions found above for the intervals in which the scalar field is constant, we obtain that in the thin wall limit we have

$$
B=C= \begin{cases}\sqrt{\frac{2 \alpha^{2}}{V_{B}}+A^{2}}, & 0<s<\bar{s} \\ b \sqrt{\frac{2 \alpha^{2}}{V_{A}}+A^{2}}, & \bar{s}<s<s_{M},\end{cases}
$$

where $b$ is a constant of integration that cannot be absorbed by redefinition of the space variables. Therefore, if we require continuity of the fields $B$ and $C$ we get 


$$
\begin{aligned}
\bar{B} & =\bar{C}=\sqrt{\frac{2 \alpha^{2}}{V_{B}}+\bar{A}^{2}}=b \sqrt{\frac{2 \alpha^{2}}{V_{A}}+\bar{A}^{2}} \\
& =\sqrt{2} \alpha b \sqrt{\left(\frac{1}{b^{2}-1}\right)\left(\frac{1}{V_{B}}-\frac{1}{V_{A}}\right)},
\end{aligned}
$$

and

$$
\bar{A}^{2}=\frac{2 \alpha^{2}}{b^{2}-1}\left(\frac{1}{V_{B}}-\frac{b^{2}}{V_{A}}\right) .
$$

Thus, we obtain in the thin wall limit

$$
\begin{aligned}
\pm \Gamma= & \pm \frac{2 \operatorname{Vol}(X) i}{\hbar}\left[\left.\sqrt{V_{B}} F_{\mathrm{III}}\left[V_{B}, A\right]\right|_{A_{0}} ^{\bar{A}}-\left.\sqrt{V_{A}} F_{\mathrm{III}}\left[V_{A}, A\right]\right|_{A_{0}} ^{\bar{A}}\right] \\
& +\frac{\operatorname{Vol}(X)}{\hbar} \bar{A}^{2}\left(\frac{2 \alpha^{2}}{V_{B}}+\bar{A}^{2}\right)^{2} T .
\end{aligned}
$$

Therefore for this metric we can also find an expression for $\Gamma$ in terms of only two variables, in this case $\bar{A}$ and $T$. Note that in this case we have $\lim _{x \rightarrow 0} F_{\text {III }}[V, x]=0$ for any value of $V$, therefore we can choose $A_{0}=0$ in the case of having both $V_{A}$ and $V_{B}$ positive (since the relations (113) hold with $B$ and $C$ being real functions).

Since the latter expression is valid for any nonzero $V_{A, B}$ we obtain finally

$$
\begin{aligned}
\pm 2 \operatorname{Re}[\Gamma]= & \mp \frac{4 \operatorname{Vol}(X)}{\hbar}\left\{\left.\operatorname{Im}\left[\sqrt{V_{B}} F_{\mathrm{III}}\left[A, V_{B}\right]\right]\right|_{A_{0}} ^{\bar{A}}\right. \\
& \left.-\operatorname{Im}\left[\left.\sqrt{V_{A}} F_{\mathrm{III}}\left[A, V_{A}\right]\right|_{A_{0}} ^{\bar{A}}\right]\right\} \\
& +\frac{2 \operatorname{Vol}(X)}{\hbar} \bar{A}^{2}\left(\frac{2 \alpha^{2}}{V_{B}}+\bar{A}^{2}\right)^{2} T .
\end{aligned}
$$

As we have pointed out before, taking the limit $\alpha \rightarrow 0$, this result simplifies to the corresponding result for the flat FLRW metric (56), therefore showing consistency of this method.

Looking for an extremum for this expression, that is looking for the points that satisfy $\frac{\partial \Gamma}{\partial \ddot{A}}=0$, we obtain that the following equation fulfils

$$
\begin{aligned}
T \bar{A} & \left(3 \bar{A}^{4}+\frac{8 \alpha^{2}}{V_{B}} \bar{A}^{2}+\frac{4 \alpha^{4}}{V_{B}^{2}}\right) \\
= & \mp i\left[\sqrt{\left(3 \bar{A}^{2} V_{B}+4 \alpha^{2}\right)\left(\bar{A}^{2}+\frac{\alpha^{2}}{V_{B}}\right)}\right. \\
& \left.-\sqrt{\left(3 \bar{A}^{2} V_{A}+4 \alpha^{2}\right)\left(\bar{A}^{2}+\frac{\alpha^{2}}{V_{A}}\right)}\right]
\end{aligned}
$$

We note that for the case in which both potentials are positive, there is only the solution $T=0$. Therefore, in this case we again does not have a minimum with a nonzero tension term for this case.

In this case for positive values for both minima of the potential, since $T$ is an independent parameter, we can study the limit $\bar{A} \rightarrow 0$ in (121), giving a vanishing result for $\Gamma$. We note that for this metric this is the only situation in which we can access this limit.

For a value of the potential equal to zero, the system of equations (7) and (10) for a constant scalar field is inconsistent. Therefore, the results found for this metric are only valid for $V_{A, B} \neq 0$. We could try another factorization of the Hamiltonian or equivalently another ordering factor but we fail to obtain a particular factorization that could lead us to both a solvable system for a zero potential and an expression for the integral in (114) that is computable.

In order to visualize what is the consequence of having an anisotropic universe for the transition probability, we plot the probability (16) for this metric using (121) with different values of $\alpha$ which measures the degree of anisotropy of the system, having in the limit $\alpha \rightarrow 0$ the isotropy limit which is the flat FLRW case with result (60). Let us choose the minus sign both in the left and in the right of (121) so we have a well behaved probability and the plot given in units such that $\frac{2 \mathrm{Vol}(X)}{\hbar}=1$ in both cases. We choose two positive values for the potential minima, namely $V_{B}=10, V_{A}=100$. Since both expressions don't have extrema, we firstly set $T=1$ and plot the probability versus $\bar{A}$, or equivalently $\bar{a}$. In Fig. 1 we present such plot, we see that a generic feature of anisotropy is to reduce the transition probability and make it fall faster as $\alpha^{2}$ increases. The graph corresponding to isotropy, or the flat FLRW case, comes only from the tension term in (60) as was explained before, whereas the contribution coming from

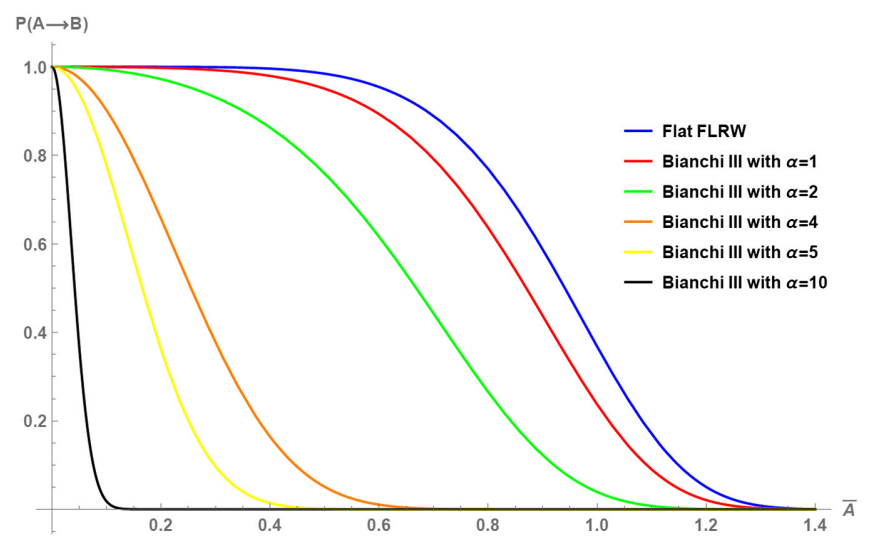

FIG. 1. Transition probability in units such that $\frac{2 \mathrm{Vol}(X)}{\hbar}=1$, with $V_{A}=100, V_{B}=10$ and $T=1$ for Bianchi III with $\alpha=10$ (black line), $\alpha=5$ (yellow line), $\alpha=4$ (orange line), $\alpha=2$ (green line), $\alpha=1$ (red line) and $\alpha=0$ or flat FLRW (blue line). The contribution comes mostly from the tension term in (121) since the other term is about 16 order of magnitudes smaller in the whole range. 
the Bianchi III cases (121) come mostly from the tension term also since for these values of the potential minima the contribution from the other term is around 16 orders of magnitude smaller in the whole range we are plotting. However, choosing different values of the parameters does not change the behavior just described. Since we have two independent variables in (121) we also plot the probability versus the tension. Choosing the same parameters as before and $\bar{A}=1$, we present this plot in Fig. 2. We see that the same behavior is encountered, namely, the probability is decreased and falls faster as $\alpha^{2}$ increases.

In order to study transitions more generally, we can also used the parameter $s$ defined in (39). In this case we obtain for the classical action

$$
S_{0}= \pm \sqrt{2 \operatorname{Vol}(X)} \int_{A_{0}, B_{0}, C_{0}, \phi_{B}}^{A_{m}, B_{m}, C_{m}, \phi_{A}} \frac{B C\left(\alpha^{2}+A^{2} V(\phi)\right)^{3 / 2}}{A \sqrt{2 V(\phi)\left(4 \alpha^{2}+3 A^{2} V(\phi)\right)-A^{2}\left(V^{\prime}(\phi)\right)^{2}}} d s
$$

where

$$
d s=\sqrt{-\frac{2}{A B} d A d B-\frac{2}{A C} d A d C-\frac{2}{B C} d B d C+d \phi^{2}} .
$$

Using the Hamiltonian constraint with (103) for this metric we obtain that the $d s$ term can be related to the kinetic term in the WDW equation as

$$
\begin{aligned}
-\frac{2}{A B} \dot{A} \dot{B}-\frac{2}{A C} \dot{A} \dot{C}-\frac{2}{B C} \dot{B} \dot{C}+\dot{\phi}^{2} & =\frac{2 N^{2}}{A^{2} B^{2} C^{2}}\left[\frac{A^{2}}{4} \pi_{A}^{2}-\frac{A B}{2} \pi_{A} \pi_{B}-\frac{A C}{2} \pi_{A} \pi_{C}+\frac{B^{2}}{4} \pi_{B}^{2}-\frac{B C}{2} \pi_{B} \pi_{C}+\frac{C^{2}}{4} \pi_{C}^{2}+\frac{1}{2} \pi_{\phi}^{2}\right] \\
& =-\frac{2 N^{2}}{A^{2}}\left(\alpha^{2}+A^{2} V(\phi)\right) .
\end{aligned}
$$

Therefore, if the integrand in (123) is real, we can have contributions for the transition probability just by having a negative kinetic term in the WDW equation, which can be achieved by having correct values for the momenta, or as the second equality of the last expression implies, just by having a positive potential. If we want to explore a system without an scalar field, the expression for the classical action does not have a well defined behavior, this happens

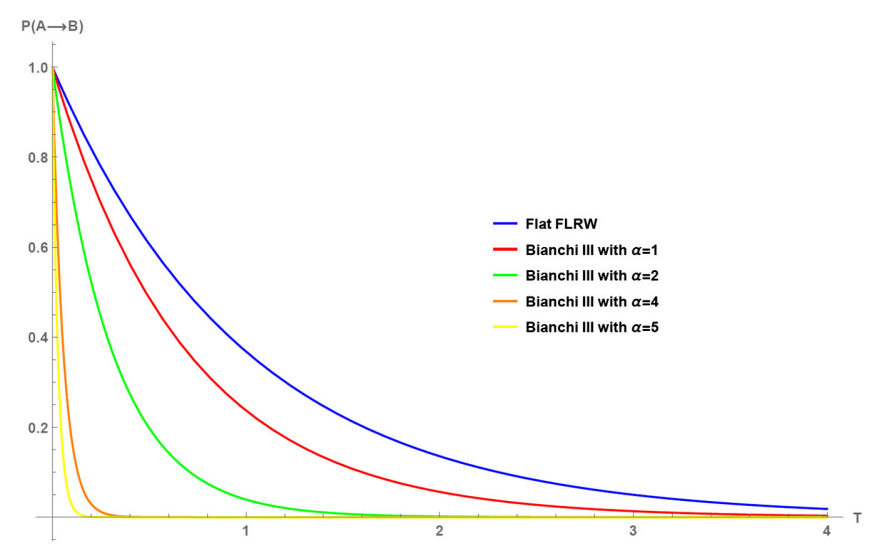

FIG. 2. Transition probability in units such that $\frac{2 \mathrm{Vol}(X)}{\hbar}=1$, with $V_{A}=100, V_{B}=10$ and $\bar{A}=1$ for Bianchi III with $\alpha=5$ (yellow line), $\alpha=4$ (orange line), $\alpha=2$ (green line), $\alpha=1$ (red line) and $\alpha=0$ or flat FLRW (blue line). because, as we have seen previously, the system of differential equations in this case is inconsistent.

\section{TRANSITIONS IN BIANCHI IX}

In this subsection we finally study the anisotropic universe described by the biaxial Bianchi IX metric [24] given by

$$
d s^{2}=-\frac{N^{2}(t)}{q(t)} d r^{2}+\frac{p(t)}{4}\left(\sigma_{1}^{2}+\sigma_{2}^{2}\right)+\frac{q(t)}{4} \sigma_{3}^{2},
$$

where

$$
\begin{aligned}
& \sigma_{1}=\sin \psi d \theta-\cos \psi \sin \theta d \omega, \\
& \sigma_{2}=\cos \psi d \theta+\sin \psi \sin \theta d \omega, \\
& \sigma_{3}=-(d \psi+\cos \theta d \omega)
\end{aligned}
$$

and $0 \leq \psi \leq 4 \pi, 0 \leq \theta \leq \pi$ and $0 \leq \omega \leq 2 \pi$. For our purposes we write down the latter expression for the metric (126) in terms of the trigonometric functions we have

$$
\begin{aligned}
d s^{2}= & -\frac{N^{2}(t)}{q} d t^{2}+\frac{q(t)}{4} d \psi^{2}+\frac{q(t)}{2} \cos \theta d \psi d \omega \\
& +\frac{p(t)}{4} d \theta^{2}+\frac{1}{4}\left(p(t) \sin ^{2} \theta+q(t) \cos ^{2} \theta\right) d \omega^{2}
\end{aligned}
$$


Now we consider the presence of a scalar field as in the previous cases, we have in this case the Lagrangian

$\mathcal{L}=\frac{1}{16}\left[-\frac{q}{2 N p} \dot{p}^{2}-\frac{\dot{q} \dot{p}}{N}-\frac{2 q}{p} N+8 N\right]+\frac{p}{8}\left[\frac{q \dot{\phi}^{2}}{2 N}-N V\right]$.

Moreover the canonical momenta are

$$
\begin{aligned}
& \pi_{N}=0, \quad \pi_{p}=-\frac{1}{16 N}\left(\frac{q}{p} \dot{p}+\dot{q}\right), \\
& \pi_{q}=-\frac{\dot{p}}{16 N}, \quad \pi_{\phi}=\frac{p q}{8 N} \dot{\phi} .
\end{aligned}
$$

Thus we obtain the Hamiltonian constraint

$$
\begin{aligned}
H & =N\left[\frac{8 q}{p} \pi_{q}^{2}-16 \pi_{p} \pi_{q}+\frac{4}{p q} \pi_{\phi}^{2}+\frac{1}{8}\left(\frac{q}{p}+p V(\phi)-4\right)\right] \\
& \approx 0 .
\end{aligned}
$$

Focusing only in the term inside brackets in the previous equation we have that in this case the coordinates in minisuperspace are $\left\{\Phi^{M}\right\}=\{q, p, \phi\}$, the nonvanishing components of the metric are

$$
G^{q q}=\frac{16 q}{p}, \quad G^{p q}=-16, \quad G^{\phi \phi}=\frac{8}{p q},
$$

and the function $f$ is given by

$$
f(p, q, \phi)=\frac{1}{8}\left(\frac{q}{p}+p V(\phi)-4\right) .
$$

We also have in this case the volume of the three dimensional slice is written as

$\operatorname{Vol}(X)=\int_{\omega=0}^{2 \pi} \int_{\theta=0}^{\pi} \int_{\psi=0}^{4 \pi} \sin \theta d \psi d \theta d \omega=16 \pi^{2}$,

which is finite because the spatial slice considered is compact.

Moreover we choose the parameter $s$ as in the previous cases, then we obtain for the classical actions

$$
\begin{aligned}
S_{0}\left(p_{0}, q_{0}, \phi_{B} ; p_{m}, q_{m}, \phi_{A}\right)= & -4 \pi^{2}\left\{\int_{0}^{\bar{s}-\delta s} \frac{d s}{C(s)}\left[\frac{q}{p}+p V_{B}-4\right]+\int_{\bar{s}-\delta s}^{\bar{s}+\delta s} \frac{d s}{C(s)}\left[\frac{q}{p}+p V(\phi)-4\right]\right. \\
& \left.+\int_{\bar{s}+\delta s}^{s_{M}} \frac{d s}{C(s)}\left[\frac{q}{p}+p V_{A}-4\right]\right\}, \\
S_{0}\left(p_{0}, q_{0}, \phi_{A} ; p_{m}, q_{m}, \phi_{A}\right)= & -4 \pi^{2} \int_{0}^{s_{M}} \frac{d s}{C(s)}\left[\frac{q}{p}+p V_{A}-4\right] .
\end{aligned}
$$

Consequently then we have

$$
\pm \Gamma=-\frac{4 \pi^{2} i}{\hbar} \int_{0}^{\bar{s}-\delta s} \frac{d s}{C(s)}\left[\frac{q}{p}+p V_{B}-4\right]+\frac{4 \pi^{2} i}{\hbar} \int_{0}^{\bar{s}-\delta s} \frac{d s}{C(s)}\left[\frac{q}{p}+p V_{A}-4\right]-\frac{4 \pi^{2} i}{\hbar} \int_{\bar{s}-\delta s}^{\bar{s}+\delta s} \frac{d s}{C(s)} p\left[V(\phi)-V_{A}\right] .
$$

In the case of a constant scalar field we obtain the solution

$$
\begin{aligned}
C^{2}(s) & =-1024 \pi^{4} \frac{3 q-2 V_{A, B} p^{2}}{p^{2}\left(q+p^{2} V_{A, B}-4 p\right)}, \\
\frac{d q}{d s} & =\frac{1}{16 \pi^{2}} \frac{\left(2 q-V_{A, B} p^{2}\right)\left(q+p^{2} V_{A, B}-4 p\right)}{3 q-2 V_{A, B} p^{2}}, \\
\frac{d p}{d s} & =-\frac{1}{16 \pi^{2}} \frac{p\left(q+p^{2} V_{A, B}-4 p\right)}{3 q-2 V_{A, B} p^{2}},
\end{aligned}
$$

whose fields are related as

$$
\frac{1}{2 q-V_{A, B} p^{2}} \frac{d q}{d s}=-\frac{1}{p} \frac{d p}{d s}
$$

That expression can be rewritten as the differential equation

$$
p d q+\left(2 q-V_{A, B} p^{2}\right) d p=0
$$

from where we obtain

$$
q=\frac{c}{p^{2}}+\frac{V_{A, B}}{4} p^{2}
$$

where $c$ is an integration constant. Since by definition both $p$ and $q$ must be positive functions, we note that we can impose the condition $\lim _{p \rightarrow 0} q=0$ in the interval we are interested in for positive values of the potential minima so we can have a spatial singularity at the beginning of the path, then 


$$
q=\frac{V_{A, B}}{4} p^{2} .
$$

$-4 \pi^{2} \int \frac{d s}{C(s)}\left[\frac{q}{p}+p V_{A, B}-4\right]= \pm 2 \pi^{2} \sqrt{5 V_{A, B}} F_{\mathrm{IX}}\left[V_{A, B}, p\right]$,

With this result we can again express the two first integrals in (137) in terms of an integral in $p$ as in the previous cases obtaining

where we have defined

$$
\begin{aligned}
F_{\mathrm{IX}}[V, x] & =\int \sqrt{x\left(\frac{5 V}{16} x-1\right)} d x \\
& =\frac{1}{4\left(\frac{5 V}{16}\right)^{3 / 2} \sqrt{x\left(\frac{5 V}{16} x-1\right)}} \times\left[\sqrt{\frac{5 V}{16}} x\left(1-\frac{15 V}{16} x+\frac{25 V^{2}}{128} x^{2}\right)-\sqrt{x\left(\frac{5 V}{16} x-1\right)} \ln \left[\frac{5 V}{16} \sqrt{x}+\sqrt{\frac{5 V}{16}} \sqrt{\left.\frac{5 V}{16} x-1\right]}\right] .\right.
\end{aligned}
$$

We can also define a tension term in this case as

$$
16 \pi^{2} \bar{p} T=-4 \pi^{2} i \int_{\bar{s}-\delta s}^{\bar{s}+\delta s} \frac{d s}{C(s)} p\left[V(\phi)-V_{A}\right] .
$$

Therefore, we obtain in the thin wall limit an expression for the decay rate

$$
\begin{aligned}
\pm \Gamma= & \pm \frac{2 \pi^{2} i \sqrt{5}}{\hbar}\left\{\left.\sqrt{V_{B}} F_{\mathrm{IX}}\left[V_{B}, p\right]\right|_{p_{0}} ^{\bar{p}}-\left.\sqrt{V_{A}} F_{\mathrm{IX}}\left[V_{A}, p\right]\right|_{p_{0}} ^{\bar{p}}\right\} \\
& +\frac{16 \pi^{2}}{\hbar} \bar{p} T,
\end{aligned}
$$

and again it is expressed in terms of only two independent variables, namely $\bar{p}$ and $T$. In this case we can safely choose in any case $p_{0}=0$. Therefore we obtain finally

$$
\begin{aligned}
\pm 2 \operatorname{Re}[\Gamma]= & \mp \frac{4 \pi^{2} \sqrt{5}}{\hbar}\left\{\left.\operatorname{Im}\left[\sqrt{V_{B}} F_{\mathrm{IX}}\left[V_{B}, p\right]\right]\right|_{0} ^{\bar{p}}\right. \\
& \left.-\left.\operatorname{Im}\left[\sqrt{V_{A}} F_{\mathrm{IX}}\left[V_{A}, p\right]\right]\right|_{0} ^{\bar{p}}\right\}+\frac{32 \pi^{2}}{\hbar} \bar{p} T,
\end{aligned}
$$

and the condition for an extremum leads to the equation $8 T=\mp \sqrt{5} i\left[\sqrt{V_{B} \bar{p}\left(\frac{5 V_{B}}{16} \bar{p}-1\right)}-\sqrt{V_{A} \bar{p}\left(\frac{5 V_{A}}{16} \bar{p}-1\right)}\right]$.

Both results are valid for any values of the potential minima $V_{A, B}$ greater than zero. For negative values the integration constant cannot be set to zero and then the integral in the expression (143) cannot be performed for an arbitrary constant. We find that in this case we have in the limit $\bar{p} \rightarrow 0$ that $T \rightarrow 0$ and $\Gamma \rightarrow 0$, so once again we can observe that the transition probability near the primordial singularity is regular.

In the case in which one of the potentials is zero, we have the solution for a constant scalar field

$$
\begin{aligned}
C^{2}(s) & =-1024 \pi^{4} \frac{3 q}{p^{2}(q-4 p)}, \\
\frac{d q}{d s} & =\frac{1}{24 \pi^{2}}(q-4 p), \\
\frac{d p}{d s} & =-\frac{1}{48 \pi^{2}} \frac{p(q-4 p)}{q},
\end{aligned}
$$

and the coordinates are related through the expression

$$
\frac{1}{q} \frac{d q}{d s}=-\frac{2}{p} \frac{d p}{d s}
$$

Then, absorbing a multiplicative constant by redefinition of the spatial variables we get

$$
q=\frac{1}{p^{2}} .
$$

With these relations we obtain

$$
-\left.4 \pi^{2} \int \frac{d s}{C(s)}\left[\frac{q}{p}+p V-4\right]\right|_{V=0}= \pm 2 \sqrt{3} \pi^{2} F_{\mathrm{IX}}^{0}(p),
$$

where we have defined

$$
\begin{aligned}
F_{\mathrm{IX}}^{0}(x) & =\int \frac{\sqrt{4 x^{3}-1}}{x^{3}} d x \\
& =-\frac{\sqrt{4 x^{3}-1}}{2 x^{2}}+\frac{i 2^{1 / 3} 3^{3 / 4}}{\sqrt{4 x^{3}-1}} \sqrt{(-1)^{5 / 6}\left(2^{2 / 3} x-1\right)} \sqrt{1+2^{2 / 3} x+2^{4 / 3} x^{2}} F\left(\arcsin \left(\frac{\sqrt{-(-1)^{5 / 6}-i 2^{2 / 3} x}}{3^{1 / 4}}\right),(-1)^{1 / 3}\right) .
\end{aligned}
$$


The only possible case now is $V_{B}=0$ and $V_{A}>0$. Then, we obtain in the limit considered

$$
\begin{aligned}
\pm \Gamma= & \pm \frac{2 \pi^{2} i}{\hbar}\left\{\left.\sqrt{3} F_{\mathrm{IX}}^{0}(p)\right|_{p_{0}} ^{\bar{p}}-\left.\sqrt{5 V_{A}} F_{\mathrm{IX}}\left[V_{A}, p\right]\right|_{p_{0}} ^{\bar{p}}\right\} \\
& +\frac{16 \pi^{2}}{\hbar} \bar{p} T .
\end{aligned}
$$

We note that $F_{\mathrm{IX}}^{0}(p)$ is divergent in the limit $p \rightarrow 0$, therefore in this case $p_{0} \neq 0$. Thus finally

$$
\begin{aligned}
\pm 2 \operatorname{Re}[\Gamma]= & \mp \frac{4 \pi^{2}}{\hbar}\left\{\left.\sqrt{3} \operatorname{Im}\left[F_{\mathrm{IX}}^{0}(p)\right]\right|_{p_{0}} ^{\bar{p}}\right. \\
& \left.-\left.\sqrt{5 V_{A}} \operatorname{Im}\left[F_{\mathrm{IX}}\left[V_{A}, p\right]\right]\right|_{p_{0}} ^{\bar{p}}\right\}+\frac{32 \pi^{2}}{\hbar} \bar{p} T .
\end{aligned}
$$

If we look for an extremum of the above expression we obtain that the following equation must hold

$$
8 T=\mp i\left[\frac{\sqrt{3\left(4 \bar{p}^{3}-1\right)}}{\bar{p}^{3}}-\sqrt{5 V_{A} \bar{p}\left(\frac{5 V_{A}}{16} \bar{p}-1\right)}\right]
$$

Since $T$ is real, the above condition leads to the equation

$$
4 \bar{p}^{3}-1=0, \quad 8 T= \pm i \sqrt{5 V_{A} \bar{p}\left(\frac{5 V_{A}}{16} \bar{p}-1\right)} .
$$

Therefore we find

$$
\bar{p}=4^{-1 / 3}, \quad T=\frac{1}{8} \sqrt{\frac{5 V_{A}}{4^{1 / 3}}\left(1-\frac{5 V_{A}}{16\left(4^{1 / 3}\right)}\right)},
$$

and consequently we get the condition $V_{A}<\frac{16\left(4^{1 / 3}\right)}{5} \approx 5.08$. Then substituting Eq. (158) back into (155) we finally obtain

$$
\begin{aligned}
\pm 2 \operatorname{Re}[\Gamma]= & 2\left\{\frac { 2 \pi ^ { 2 } } { \hbar } \left[\left.\sqrt{3} \operatorname{Im}\left[F_{\mathrm{IX}}^{0}(p)\right]\right|_{p_{0}} ^{4^{-1 / 3}}\right.\right. \\
& \left.-\left.\sqrt{5 V_{A}} \operatorname{Im}\left[F_{\mathrm{IX}}\left[V_{A}, p\right]\right]\right|_{p_{0}} ^{4^{-1 / 3}}\right] \\
& \left.+\frac{\sqrt{5 V_{A}} \pi^{2}}{\hbar} \sqrt{1-\frac{5 V_{A}}{16\left(4^{1 / 3}\right)}}\right\} .
\end{aligned}
$$

If the last condition does not hold, then the expression for $\pm \Gamma$ does not have a minimum with a nonvanishing tension term.

Finally, to study transitions more generally, we can use the parameter $s$ defined in (39). In this case we obtain for the classical action
$S_{0}= \pm \frac{\pi}{2} \int_{q_{0}, p_{0}, \phi_{B}}^{q_{m}, p_{m}, \phi_{A}} \frac{\left(q+p^{2} V(\phi)-4 p\right)^{3 / 2}}{\sqrt{2 p^{2} V(\phi)-3 q-\frac{p^{4}}{2 q}\left(V^{\prime}(\phi)\right)^{2}}} d s$,

where

$$
d s=\sqrt{-\frac{1}{8} d q d p-\frac{q}{16 p} d p^{2}+\frac{p q}{8} d \phi^{2}} .
$$

Using the Hamiltonian constraint (131) for this metric we obtain

$$
\begin{aligned}
-\frac{\dot{q} \dot{p}}{8}-\frac{q}{16 p} \dot{p}^{2}+\frac{p q}{8} \dot{\phi}^{2} & =2 N^{2}\left[\frac{8 q}{p} \pi_{q}^{2}-16 \pi_{p} \pi_{q}+\frac{4}{q p} \pi_{\phi}^{2}\right] \\
& =-\frac{N^{2}}{4}\left(\frac{q}{p}+p V(\phi)-4\right)
\end{aligned}
$$

Therefore, if the integrand in (160) is real, we can have contributions for the transition probability just by having a negative kinetic term in the WDW equation. It may seem from the first equality that if we remove the scalar field, we could have a negative term contributing to the probability. However, if we remove the scalar field, we obtain the action

$$
\begin{aligned}
& \left.S_{0}\right|_{\phi=0, V=0} \\
& \quad= \pm \frac{\pi}{2} \int_{q_{0}, p_{0}}^{q_{m}, p_{m}}(q-4 p) \sqrt{\frac{4 p-q}{3 q}} \sqrt{-\frac{1}{8} d q d p-\frac{q}{16 p} d p^{2}},
\end{aligned}
$$

but from (162) we obtain

$$
-\frac{\dot{q} \dot{p}}{8}-\frac{q}{16 p} \dot{p}^{2}=\frac{N^{2}}{4}\left(\frac{4 p-q}{p}\right) .
$$

We note from these last expressions that it is not possible to have an imaginary term coming from the action.

\section{FINAL REMARKS}

In the present article we have described a general procedure that applies the WKB approximation to quantum gravity encoded in the WDW equation with a Lorentzian perspective. This procedure was proposed originally in [11] in the computation of transition probabilities for vacuum decay in the presence of a scalar potential.

Moreover we have presented explicit computations of the transition probabilities in the thin wall limit for three homogeneous but anisotropic metrics, as well as for the isotropic metric FLRW with both positive and null curvature for comparative purposes. For the FLRW metric with positive curvature, we reproduced the same result obtained 
in Ref. [11]. However in our proposal we have a slight variation which allow us to obtain a system of equations which is solvable and it does not require us to propose a particular path in field space, which has the advantage of obtaining an unique possible result.

For both anisotropic and isotropic metrics we found analytic expressions for the logarithm of the transition probabilities, which depends on the potential minima and two variables, namely the tension term and one coming from the metric. For the anisotropic metrics we found that a consequence of the semiclassical approximation is that in all cases the metric degrees of freedoms are related, therefore reducing to one the degree of freedom needed to compute the classical action, this is similar to the procedure used in [21] in which the degrees of freedom are also reduced in order to compute the classical action. The last step is to look for an extremum of the logarithm of the transition probability.

In the case of FLRW with positive curvature it was possible to do it obtaining at the end the transition probability as a function of only the potential minima and the scale factor. However for all other metrics this step was found to be troublesome. Since the existence of such a minimum was found to be in general highly dependent on the specific values of the potential minima. Thus they all have an additional variable in its final result.

For example, for the flat FLRW, Kantowski-Sachs and Bianchi III metrics it was found that no minimum exists with a nonzero tension if both potential minima are positive, that is for transition from dS to dS. For Bianchi IX with positive potential minima the existence of a minimum depends completely on the specific values of the potential in such points. For Bianchi IX with the true vacuum at zero potential, it was found that the existence of a minimum leads to a condition for the false vacuum potential and concrete values for the tension term and the one coming for the metric, therefore having one less term that FLRW with positive curvature.

For the Bianchi III metric we plot the transition probability as a function of the degree of freedom coming from the metric, which was one of the scale factors, as well as a function of the tension. We see that we recover the flat FLRW metric result taking the isotropic limit and we show that the degree of anisotropy leads in general to a smaller probability and a faster decay in its plot as anisotropy is increased. This result coincides completely with the general behavior described in [22] for the Bianchi I model using a different approach.
Although in this work we used the WKB approximation based on GR, we obtain that many of the expressions found for the transition probabilities are well behaved and represented by regular functions in the ultraviolet limit. Thus we consider them reliable physical results and it is worth studying these processes in an early universe in despite that GR is nonrenormalizable. In particular, we found that the probabilities for the FLRW metrics and the Kantowski-Sachs metric are well behaved for all minima of the potential different from zero. For the Bianchi III and Bianchi IX metrics this was possible only for positive values of the minima of the potential. In any possible case, all probabilities goes to 1 in this limit. We interpret this result as follows: the closer we are from the initial singularity the more probable these transitions are to occur. The probability will decrease as soon as we move away from the singularity. However, certainly in order to study the behavior near the big bang singularity it is necessary to use a gravity theory proposal with a better behavior near the singularity than GR. It would be interesting to study theories of this type as string theory or Horava-Lifshitz theory in order to check if the WKB approximation to quantum gravity, proposed $[10,11]$ and used in the present article, can be implemented in these theories. At least for Horava-Lifshitz theory [25] which is very similar to GR in the infrared and whose Einstein-Hilbert action is corrected with higher-order terms in the spatial curvature in the ultraviolet, it seems to be possible to apply the mentioned WKB formalism. Some of these considerations will be reported in a forthcoming work.

Finally it is worth mentioning that in the examples of anisotropic metrics we considered here, the only one with a finite volume is the Bianchi IX type. Thus, in this case it is also valid the result obtained in Ref. [11], which asserts that the universe will remain closed after a transition for anisotropic universes. Therefore in this context the transition also respects the closeness of the spatial universe. Thus this certainly extends the debate landscape/swampland corresponding to open/closed universes [26-29] to the anisotropic context.

\section{ACKNOWLEDGMENTS}

It is a pleasure to thank Professor F. Quevedo for comments on the manuscript. D. M.-P. would also like to thank CONACyT for a Grant No. 784617. 
[1] R. L. Arnowitt, S. Deser, and C. W. Misner, The dynamics of general relativity, Gen. Relativ. Gravit. 40, 1997 (2008).

[2] J.A. Wheeler, Superspace and the nature of quantum geometrodynamics, Topics in Nonlinear Physics, edited by N. J. Zabusky (Springer-Verlag NY, Inc., 1968), pp. 615-724.

[3] B.S. DeWitt, Quantum theory of gravity I, The canonical theory, Phys. Rev. 160, 1113 (1967).

[4] S. R. Coleman, The fate of the false vacuum. 1. Semiclassical theory, Phys. Rev. D 15, 2929 (1977); 16, 1248(E) (1977).

[5] C. G. Callan, Jr. and S. R. Coleman, The fate of the false vacuum. 2. First quantum corrections, Phys. Rev. D 16, 1762 (1977).

[6] S. R. Coleman and F. De Luccia, Gravitational effects on and of vacuum decay, Phys. Rev. D 21, 3305 (1980).

[7] S. J. Parke, Gravity, the decay of the false vacuum and the new inflationary universe scenario, Phys. Lett. 121B, 313 (1983).

[8] W. Fischler, D. Morgan, and J. Polchinski, Quantum nucleation of false vacuum bubbles, Phys. Rev. D 41, 2638 (1990).

[9] W. Fischler, D. Morgan, and J. Polchinski, Quantization of false vacuum bubbles: A Hamiltonian treatment of gravitational tunneling, Phys. Rev. D 42, 4042 (1990).

[10] S. P. De Alwis, F. Muia, V. Pasquarella, and F. Quevedo, Quantum transitions between Minkowski and de Sitter spacetimes, Fortschr. Phys. 68, 2000069 (2020).

[11] S. Cespedes, S. P. de Alwis, F. Muia, and F. Quevedo, Lorentzian vacuum transitions: Open or closed universes?, Phys. Rev. D 104, 026013 (2021).

[12] D. Saadeh, S. M. Feeney, A. Pontzen, H. V. Peiris, and J. D. McEwen, How Isotropic is the Universe?, Phys. Rev. Lett. 117, 131302 (2016).

[13] J. Colin, R. Mohayaee, M. Rameez, and S. Sarkar, Evidence for anisotropy of cosmic acceleration, Astron. Astrophys. 631, L13 (2019).

[14] J. Soltis, A. Farahi, D. Huterer, and C. M. Liberato, PercentLevel Test of Isotropic Expansion Using Type Ia Supernovae, Phys. Rev. Lett. 122, 091301 (2019).

[15] K. Migkas, G. Schellenberger, T. H. Reiprich, F. Pacaud, M.E. Ramos-Ceja, and L. Lovisari, Probing cosmic isotropy with a new X-ray galaxy cluster sample through the $L_{\mathrm{X}}-T$ scaling relation, Astron. Astrophys. 636, A15 (2020).

[16] K. Migkas, F. Pacaud, G. Schellenberger, J. Erler, N. T. Nguyen-Dang, T. H. Reiprich, M. E. Ramos-Ceja, and L. Lovisari, Cosmological implications of the anisotropy of ten galaxy cluster scaling relations, Astron. Astrophys. 649, A151 (2021).

[17] C. Krishnan, R. Mohayaee, E. Ó. Colgáin, M. M. SheikhJabbari, and L. Yin, Does Hubble tension signal a breakdown in FLRW cosmology?, Classical Quantum Gravity 38, 184001 (2021).

[18] C. Krishnan, R. Mohayaee, E. Ó Colgáin, M. M. SheikhJabbari, and L. Yin, Hints of FLRW breakdown from supernovae, arXiv:2106.02532.

[19] S. del Campo and A. Vilenkin, Tunneling wave function for anisotropic universe, Phys. Lett. B 224, 45 (1989).

[20] L. G. Jensen and P. J. Ruback, Bubble formation in anisotropic cosmologies, Nucl. Phys. B325, 660 (1989).

[21] V. N. Folomeev, V. T. Gurovich, and I. V. Tokareva, Generation of the scalar field and anisotropy at quantum creation of the closed universe, Nuovo Cimento Soc. Ital. Fis. 115B, 1091 (2000).

[22] R. Mansouri and M. Mohazzab, Tunneling in anisotropic cosmological models, Classical Quantum Gravity 10, 1353 (1993).

[23] C. W. Misner, Minisuperspace, in Magic Without Magic, edited by J. R. Klauder, A collection in honor of his 60th birthday (W.H. Freeman, San Francisco, 1972), pp. 441473.

[24] M. Ryan, Hamiltonian Cosmology (Springer Verlag, Berlin, 1972).

[25] P. Hořava, Quantum gravity at a Lifshitz point, Phys. Rev. D 79, 084008 (2009).

[26] B. Freivogel, M. Kleban, M. R. Martinez, and L. Susskind, Observational consequences of a landscape, J. High Energy Phys. 03 (2006) 039.

[27] M. Kleban and M. Schillo, Spatial curvature falsifies eternal inflation, J. Cosmol. Astropart. Phys. 06 (2012) 029.

[28] J. Hartle and T. Hertog, Anthropic bounds on $\Lambda$ from the no-boundary quantum state, Phys. Rev. D 88, 123516 (2013).

[29] S. W. Hawking and T. Hertog, A smooth exit from eternal inflation?, J. High Energy Phys. 04 (2018) 147. 